ARTICLE

\title{
Parabrachial nucleus circuit governs neuropathic pain-like behavior
}

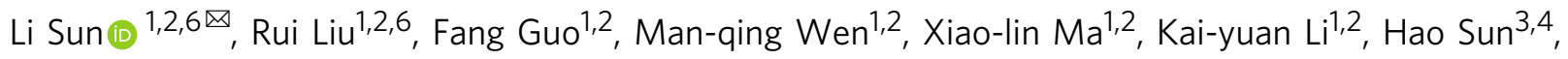
Ceng-lin Xu (1) ${ }^{1}$, Yuan-yuan $\mathrm{Li}^{1,2}$, Meng-yin $\mathrm{Wu}^{5}$, Zheng-gang Zhu (1) ${ }^{1,2}$, Xin-jian Li ${ }^{3}$, Yan-qin Yu ${ }^{1,2}$,

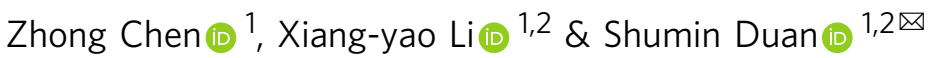

The lateral parabrachial nucleus (LPBN) is known to relay noxious information to the amygdala for processing affective responses. However, it is unclear whether the LPBN actively processes neuropathic pain characterized by persistent hyperalgesia with aversive emotional responses. Here we report that neuropathic pain-like hypersensitivity induced by common peroneal nerve (CPN) ligation increases nociceptive stimulation-induced responses in glutamatergic LPBN neurons. Optogenetic activation of GABAergic LPBN neurons does not affect basal nociception, but alleviates neuropathic pain-like behavior. Optogenetic activation of glutamatergic or inhibition of GABAergic LPBN neurons induces neuropathic pain-like behavior in naïve mice. Inhibition of glutamatergic LPBN neurons alleviates both basal nociception and neuropathic pain-like hypersensitivity. Repetitive pharmacogenetic activation of glutamatergic or GABAergic LPBN neurons respectively mimics or prevents the development of CPN ligation-induced neuropathic pain-like hypersensitivity. These findings indicate that a delicate balance between excitatory and inhibitory LPBN neuronal activity governs the development and maintenance of neuropathic pain.

\footnotetext{
${ }^{1}$ Department of Neurobiology and Department of Neurology of Second Affiliated Hospital, Zhejiang University School of Medicine, 310058 Hangzhou, China. ${ }^{2}$ Research Units for Emotion and Emotion Disorders, NHC and CAMS Key Laboratory of Medical Neurobiology, MOE Frontier Science Center for Brain Research and Brain-Machine Integration, School of Brain Science and Brain Medicine, Zhejiang University, 310058 Hangzhou, China. ${ }^{3}$ Department of Neurology of the Second Affiliated Hospital, Interdisciplinary Institute of Neuroscience and Technology, Zhejiang University School of Medicine, 310020 Hangzhou, China. ${ }^{4}$ Key Laboratory of Biomedical Engineering of Ministry of Education, College of Biomedical Engineering and Instrument Science, Zhejiang University, 310027 Hangzhou, China. ${ }^{5}$ Department of Epidemiology and Biostatistics, School of Public Health, Zhejiang University, 310058 Hangzhou, China.

${ }^{6}$ These authors contributed equally: Li Sun, Rui Liu. ${ }^{凶}$ email: sunli2014@zju.edu.cn; duanshumin@zju.edu.cn
} 
$\mathrm{N}$ europathic pain is a chronic condition caused by lesions of the peripheral or central nervous system, due to such factors as trauma, tumor invasion, stroke, diabetes, or multiple sclerosis ${ }^{1,2}$. The hallmarks of neuropathic pain include persistent hyperalgesia, allodynia, and affective responses due to sensitization of the somatosensory nervous system so that both noxious and innocuous stimuli are pathologically amplified ${ }^{3,4}$. Chronic pain can persist after the initial injury has healed and thus has been associated with plastic changes in the structure and function of the pain transmission pathway ${ }^{5,6}$, particularly at the levels of primary afferents and the spinal cord ${ }^{4,7-9}$. In addition, the gate control theory of pain, which proposes that spinal nociceptive transmission neurons are gated by inhibitory interneurons in the dorsal horn ${ }^{10}$, has also been applied to explain neuropathic pain. That is, peripheral injury may induce a dysfunction of inhibitory dorsal horn neurons, leading to sensitization and increased excitability of projection neurons ${ }^{11,12}$. However, neuropathic pain also occurs in CNS injury or brain disorders ${ }^{1,2}$, suggesting that higher brain regions may also play critical roles in neuropathic pain development. Indeed, the supraspinal and cortical areas including the primary somatosensory cortex and the anterior cingulate cortex have been also reported to be involved in the neuropathic pain ${ }^{3,4,7-9,13}$.

Two ascending pathways have been described for pain transmission to higher centers. The spinothalamic tract carries sensory inputs from spinal neurons to the thalamus, which relays the information to the somatosensory cortex for processing the sensory and discriminative aspects of pain, whereas the spinoparabrachial tract transmits the pain signal to the parabrachial nucleus $(\mathrm{PBN})^{7,14-19}$, which is reported to project to the amygdala and other brain regions to transform the sensory signals into the aversive and emotional components of pain ${ }^{20-29}$. Furthermore, the $\mathrm{PBN}$ has been associated with other important sensory processes, such as itch and craniofacial affective pain, as well as aversive emotional behaviors, including aversive learning, avoidance behavior, and anorexia/hunger ${ }^{30-36}$. However, the role of PBN in the development and maintenance of neuropathic pain, a persistent pain characterized by prominent emotional responses ${ }^{11,37,38}$, is not clear.

Using common peroneal nerve $(\mathrm{CPN})$ ligation $^{39}$ as a neuropathic pain model combined with $\mathrm{Ca}^{2+}$ signal imaging ${ }^{40}$, an optogenetics approach ${ }^{41}$, and behavioral testing $20,42,43$, we found that glutamatergic neurons in the lateral PBN (LPBN) are responsible not only for relaying basal nociception, but also for the processing of neuropathic pain. On the other hand, GABAergic neurons, which constitute only $10.1 \%$ of LPBN neurons, are not involved in the transmission of basal nociception signals. However, the activity of GABAergic LPBN neurons plays a gating role in the development and transmission of neuropathic pain-like hypersensitivity. Trans-synaptic virus tracing and electrophysiological studies identified direct synaptic innervation of and functional inhibition on glutamatergic LPBN neurons by local GABAergic neurons. These results, together with the finding that CPN ligation selectively activated glutamatergic LPBN neurons indicate that GABAergic LPBN neurons gate the sensitization of glutamatergic neurons, which is necessary and sufficient for the development and transmission of neuropathic pain.

\section{Results}

Increased activity of glutamatergic LPBN neurons in neuropathic pain-like hypersensitivity. Successful induction of neuropathic pain-like hypersensitivity was confirmed seven days after $\mathrm{CPN}$ ligation by a marked reduction in the paw withdrawal threshold (PWT) upon stimulation of the lateral aspect of the plantar paw surface by a von Frey filament (Supplementary
Fig. 1a-c). Immunostaining of c-Fos one week after CPN ligation showed a dramatic increase in the density of c-Fos-positive cells co-expressing $\mathrm{Ca}^{2+} /$ calmodulin-dependent protein kinase IIa (CaMKIIa, a marker of glutamatergic neurons) in the LPBN as compared with Sham operation, whereas no significant difference was found between CPN-treated and Sham-treated mice in c-Fospositive cells co-expressing glutamic acid decarboxylase 67 (GAD67, a marker of GABAergic neurons) (Fig. 1a-d). Thus, after CPN ligation, $\sim 41.8 \%$ of CaMKIIa neurons were activated (co-localized with c-Fos) as compared with $\sim 10.3 \%$ in Shamtreated mice, and only $\sim 8.5 \%$ of GAD $67^{+}$neurons co-expressed c-Fos as compared with $\sim 6.3 \%$ in Sham-treated mice (Fig. 1d).

We targeted glutamatergic neurons expressing vesicular glutamate transporter 2 (VgluT2) by local injection of adeno-associated viruses (AAVs) containing a Cre-dependent eYFP tag into the LPBN of a mouse line expressing Cre recombinase under the control of the VgluT2 promoter (VgluT2-ires-Cre). We found that $\sim 83 \%$ of CaMKIIa-positive neurons in the superior LPBN (PBsl) overlapped with VgluT2 and $\sim 82.1 \%$ of CaMKIIa-positive neurons in the dorsal LPBN (PBdl) overlapped with VgluT2. On the other hand, $\sim 78 \%$ of VgluT2 ${ }^{+}$neurons in the PBsl overlapped with CaMKIIa and $\sim 76.8 \%$ of VgluT2 ${ }^{+}$neurons in the PBdl overlapped with CaMKIIa (Fig. 1e-f), suggesting that CaMKIIa ${ }^{+}$and VgluT2 ${ }^{+}$ neurons overlap extensively in the LPBN. To estimate the ratio of GABAergic/glutamatergic neurons in the LPBN, we applied fluorescence in situ hybridization using GAD1 and Slc17a6 (VgluT2) probes (Fig. 1g). We found that the ratio of GABAergic neurons (GAD1-positive cells/GAD1-positive cells + VgluT2-positive cells) was only $10.1 \%$ (Fig. $1 \mathrm{~h}$ ), consistent with the data shown in the Allen Brain Atlas (http://www.brain-map.org).

The above results indicate that glutamatergic, but not GABAergic neurons in the LPBN are activated during the development of neuropathic pain-like hypersensitivity. To investigate changes in the dynamic activity of LPBN neurons associated with neuropathic pain-like hypersensitivity, we used in vivo fiber photometry ${ }^{40}$ to monitor their $\mathrm{Ca}^{2+}$ levels. We thus injected AAVs encoding Cre-dependent driven eYFP or GCaMP7s, a genetically-encoded fluorescent $\mathrm{Ca}^{2+}$ indicator, into the LPBN of VgluT2-ires-Cre (Fig. 1i) or GAD2-ires-Cre mice (Fig. 11). Three weeks after virus injection, mice were subjected to CPN ligation or Sham operation. We found that a noxious pinch induced a small but significant $\mathrm{Ca}^{2+}$ elevation in both glutamatergic (Fig. 1j, k) and GABAergic neurons (Fig. 1m, n) in the LPBN in Sham-operated mice. However, in CPN-ligated mice the same stimulus induced dramatic $\mathrm{Ca}^{2+}$ elevation in glutamatergic (Fig. 1j, k) but not in GABAergic neurons (Fig. 1m, $\mathrm{n})$, confirming that glutamatergic LPBN neurons are selectively activated by the noxious stimulus.

To further characterize the $\mathrm{Ca}^{2+}$ signals in glutamatergic LPBN neurons associated with neuropathic pain-like hypersensitivity, we used in vivo miniaturized microscopy, which has high sensitivity and spatial resolution so that weak $\mathrm{Ca}^{2+}$ responses in individual neurons can be detected ${ }^{44,45}$. Three weeks after virus injection, activity of glutamatergic LPBN neurons in intact freelymoving mice was assessed with pinch or von Frey stimulation (pre-CPN) (Supplementary Fig. 2a-c). Subsequently, VgluT2ires-Cre mice were subjected to CPN surgery. We found that a noxious stimulus (pinch) and a strong mechanical stimulus $(1.0 \mathrm{~g}$ von Frey) of the hindpaw elicited detectable $\mathrm{Ca}^{2+}$ responses in control mice (pre-CPN) and such responses were significantly higher one week after CPN surgery (post-CPN) than pre-CPN in the same mice (Supplementary Fig. 2d, e). Interestingly, a weak mechanical stimulus ( $0.4 \mathrm{~g}$ von Frey) induced an apparent $\mathrm{Ca}^{2+}$ response in post-CPN but not in pre-CPN mice (Supplementary Fig. 2f, g), consistent with the mechanical allodynia of neuropathic pain. 
a

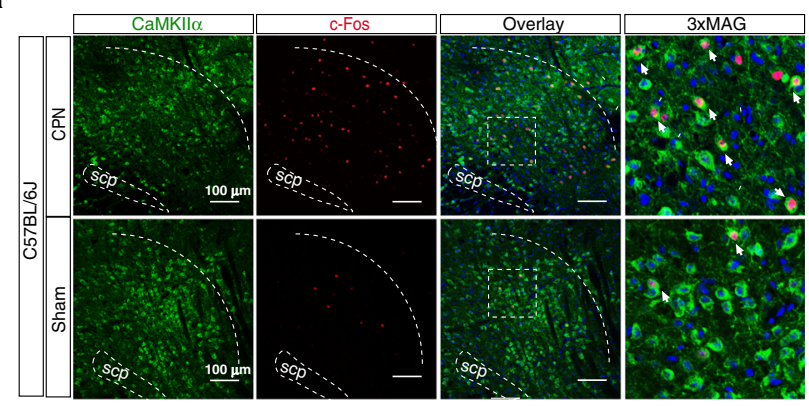

b

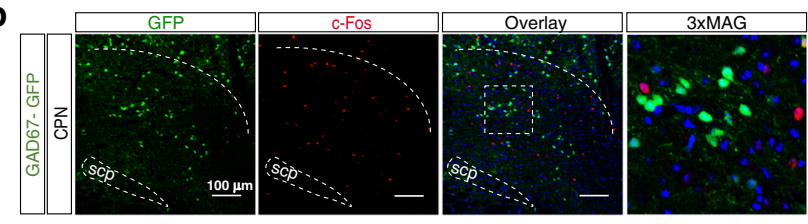

e
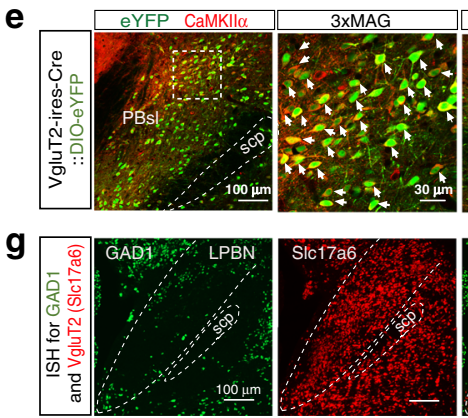

i

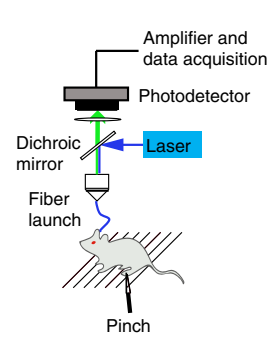

VgluT2-ires-cre mice

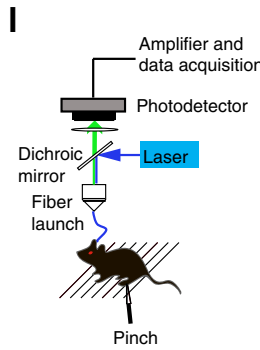

GAD2-ires-cre mice
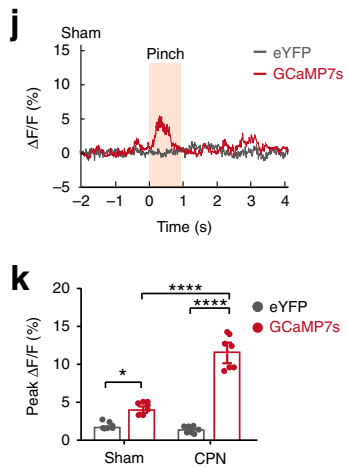

$\mathbf{m}_{\text {Sham }}$
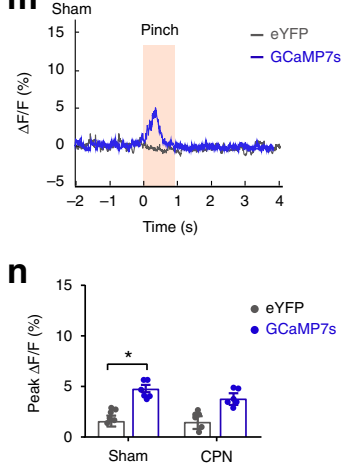
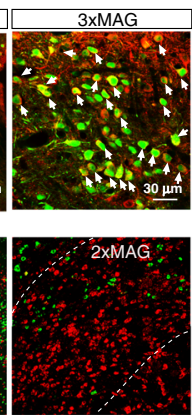

C
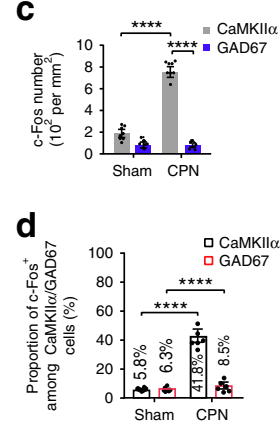

f
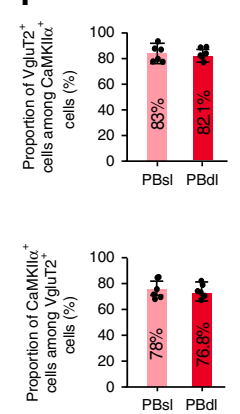

$\mathbf{h}$

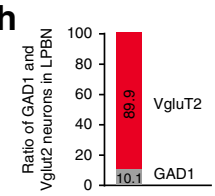

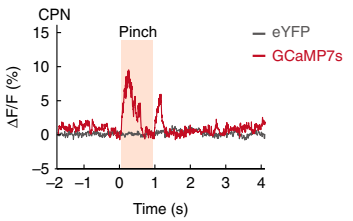
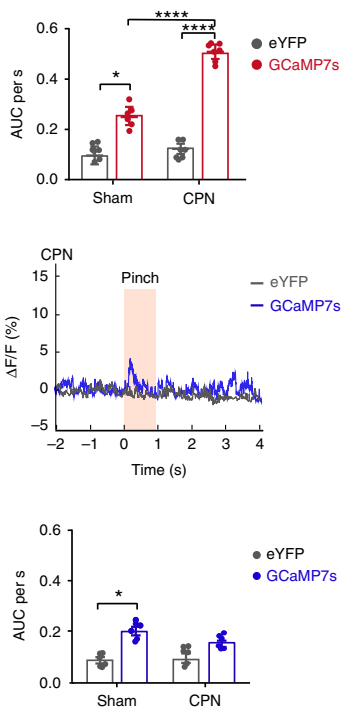

Activation of glutamatergic LPBN neurons is sufficient to induce allodynia, hyperalgesia, and the place-avoidance response. Because glutamatergic LPBN neurons were selectively activated in neuropathic pain-like hypersensitivity, we next determined whether the direct optogenetic activation of these neurons is sufficient to induce allodynia and hyperalgesia, hallmarks of neuropathic pain. We targeted the glutamatergic neurons by injection of AAVs delivering a construct that contained Cre-dependent channelrhodopsin-2 (ChR2) coupled to an eYFP tag into the LPBN of VgluT2-ires-Cre mice (Fig. 2a, c). Mice injected with AAVs containing only the eYFP tag served as controls. Current-clamp recordings from brain slices showed that blue 
Fig. 1 CPN ligation activates glutamatergic LPBN neurons. a Representative images of co-labeling (arrowheads) of CaMKIl $\alpha$-positive neurons and c-Fos immunoreactivity in the LPBN (white dashed lines). Rightmost panels, 3 times magnification of the boxed areas in the left panels. Scale bars, $100 \mu$ m. b Example images showing c-Fos positive (red) and GAD67-GFP-expressing cells in the LPBN one week after CPN ligation. Rightmost panel, 3 times magnification of the boxed area in the left panel. Blue, DAPI staining. Scale bars, $100 \mu \mathrm{m}$. c Density of c-Fos-positive cells in CaMKIll $\alpha$-expressing and GAD67-GFP-expressing cells in the LPBN. d Proportions of CaMKIl $\alpha$ or GAD67-GFP-positive cells co-expressed with c-Fos in the LPBN. e Representative images of neurons expressing VgluT2-eYFP co-localize (arrows) with the CaMKIl $\alpha$ in PBsl (left two panels) and PBdl (right two panels). 3xMAG (the second and fourth panels), 3 times magnification of the boxed area in the first and the third panels, respectively. Scale bars, $100 \mu \mathrm{m}$ and $30 \mu \mathrm{m}$ ( $3 \times \mathrm{xAG}$ ). f Percentage of CaMKIl $\alpha$-positive neurons that co-express VgluT2 (upper) and percentage of VgluT2-positive neurons that co-express CaMKIl $\alpha$ (lower). $n=898$ (PBsl) and 856 (PBdl) neurons from 5 sections from 5 mice. $\mathbf{g}$ Representative in situ hybridization for the GAD1 (green) and VgluT2 (red, Slc17a6) in LPBN. Scale bars, $100 \mu \mathrm{m}$. Rightmost panels, $2 \times$ magnification of the boxed area in the left panel. $\mathbf{h}$ Ratio of Vglut2 or GAD1-positive neurons in the LPBN as in $\mathbf{g}$. $n=6$ sections from 6 mice. i, I Schematic of the recording system for the $\mathrm{Ca}^{2+}$ signal in LPBN neurons with fiber photometry in VgluT2-ires-Cre mice (i) and GAD2-ires-Cre mice (I). $\mathbf{j}, \mathbf{m}$ Representative fluorescence signals $(\Delta F / F$ ) of GCaMP7s (red) and eYFP (dark gray) recorded from glutamatergic (j) or GABAergic (m) LPBN neurons transfected with DIO-GCaMP7s or DIO-eYFP aligned to the pinch stimulation. $\mathbf{k}, \mathbf{n}$ Averaged peak $\Delta F / F$ (left) and area under the curve (AUC) per second (right) of GCaMP7s and eYFP fluorescence signals from glutamatergic (k) or GABAergic (n) LPBN neurons. All data are presented as mean \pm s.e.m. and error bars represent s.e.m. ${ }^{\star} P<0.05$ and ${ }^{\star \star \star \star} P<0.0001$. See also Supplementary Table 1 for further statistical information. Source data are provided as a Source Data file.

laser pulses induced time-locked action potential firing in ChR2eYFP-infected neurons in the LPBN (LPBNVgluT2) (Fig. 2b), establishing the expression and function of ChR2 in these neurons. Consequently, a dramatic increase in c-Fos expression was found in LPBN after light stimulation of the LPBNVgluT2 neurons (Supplementary Fig. 3a, b). The Von Frey and Hargreaves tests were used to assess the mechanical threshold and thermal response latency of the paw-withdrawal response ${ }^{43,46}$. We found that the optical activation of LPBN VgluT2 neurons expressing ChR2-eYFP, but not those expressing eYFP, with blue laser light $(473 \mathrm{~nm}, 20 \mathrm{~Hz}, 5 \mathrm{~mW})$ induced marked mechanical allodynia, as manifested by a drastic decrease in the threshold for the pawwithdrawal response induced by von Frey filaments in Shamoperated mice (Fig. 2d). Moreover, photoactivation of LPBN VgluT2 neurons expressing ChR2-eYFP, but not those expressing eYFP, also induced thermal hyperalgesia in Sham-operated mice, as manifested by a significant decrease in the latency of the pawwithdraw response to thermal stimulation in the Hargreaves test (Fig. 2e). Similarly, illumination of LPBNCaMKIIa neurons expressing ChR2-mCherrry, but not those expressing mCherry, also induced both mechanical allodynia and thermal hyperalgesia in Sham-operated mice (Fig. 3a-c). Interestingly, this lightinduced mechanical and thermal hyperalgesia was time-locked to the light stimulation, since both the mechanical threshold and thermal latency of paw withdrawal responses returned to the control level after the stimulation (Fig. 3b, c). The lightinduced activation of LPBNCAMKII $\alpha$ neurons was confirmed by assessing the c-Fos expression level in the LPBN (Supplementary Fig. 3c, d).

Next, we used the real-time place avoidance (RTPA) assay 47,48 to determine whether glutamatergic LPBN neurons are involved in the affective component of pain behavior. Mice were habituated and placed in a two-chamber arena. The time spent in free exploration in each chamber was recorded for $10 \mathrm{~min}$ (baseline), followed by a 10 -min recording during conditioned photostimulation $(20 \mathrm{~Hz}, 5-\mathrm{ms}$ pulse width, $5 \mathrm{mV})$ of LPBNVgluT2 or LPBNCaMKIIa neurons when the mouse was in its preferred chamber, and followed again by a 10-min recording after photostimulation (Fig. 2f). As expected, upon optogenetic activation of the LPBNVgluT2 or LPBNCaMKIIa neurons, mice tended to flee to the opposite chamber, and subsequently they moved less and spent significantly more time in the unstimulated chamber, a behavior that persisted even during the 10-min post-photostimulation period (Fig. 2i, j and Fig. 3e), whereas illumination had no effect on the RTPA test in control DIO-eYFP or CaMKIIa-mCherry mice (Fig. 2g, h and Fig. 3d). On the other hand, 5 min optogenetic activation of LPBNCaMKIIa neurons did not affect the total distance moved and the ratio of time spent in the periphery and center of the open field in naïve wild-type mice (Fig. $3 \mathrm{f}-\mathrm{h}$ ), indicating that the locomotor and baseline anxiety levels were not altered by optogenetic manipulation. Altogether, these data demonstrated that the optogenetic activation of glutamatergic LPBN neurons is sufficient to induce both hyperalgesia and affective aversion in naïve mice, mimicking the typical symptoms of neuropathic pain. Although chronic pain and anxiety are often comorbid, probably due to long-term plasticity changes in some brain structures ${ }^{9,49}$, our results indicate that short-term stimulation of LPBN neurons is not sufficient to induce anxiety behavior.

Inactivation of glutamatergic LPBN neurons alleviates both basal nociception and neuropathic pain-like hypersensitivity. To further illustrate the correlation between glutamatergic neuronal activity in the LPBN and neuropathic pain-like hypersensitivity, we injected AAV-CaMKIIa-eNpHR3.0-mCherry virus bilaterally into the LPBN of wild-type mice or AAV-DIO-eNpHReYFP virus into the LPBN of VgluT2-ires-Cre mice to selectively silence the activity of LPBNCaMKIIa or LPBNVgluT2 neurons, respectively, during illumination by a 589-nm laser (Figs. 4a, 5a). We found that the eNpHR-mediated photosilencing of LPBNCaMKIIa or LPBNVgluT2 neurons drastically elevated the PWT in the von Frey test in both Sham-operated and CPN-ligated mice (Figs. 4b, 5b). An increase in paw-withdraw latency in response to thermal stimulation in the Hargreaves test also occurred in Sham-operated and CPN-ligated mice upon photosilencing of LPBNCaMKIIa or LPBN VgluT2 neurons (Figs. 4c, 5c). These results indicate that glutamatergic LPBN neurons mediate both basal nociception and neuropathic pain-like hypersensitivity.

To estimate the aversive quality of ongoing or tonic pain, we next tested real-time place preference (RTPP), which is associated with the seeking of pain relief $f^{50,51,52}$. We used this assay to determine whether photosilencing of LPBNCaMKIIa or LPBNVgluT2 neurons after CPN ligation elicits pain-reliefseeking behavior. Each mouse was first habituated to the two connected chambers for $10 \mathrm{~min}$, paired with light stimulation in one chamber and no light in the opposite chamber for another 10 min, and again no light stimulation for $10 \mathrm{~min}$ (Fig. 4d). Strikingly, we found that photoinhibition of the LPBNCaMKIIa or LPBNVgluT2 neurons significantly increased the time spent in the chamber paired with light stimulation during the light and post-light stimulation periods in $\mathrm{CPN}$-ligated mice but not in Sham-operated mice (Fig. 4e, f, g and Fig. 5d-f). Importantly, we 
a

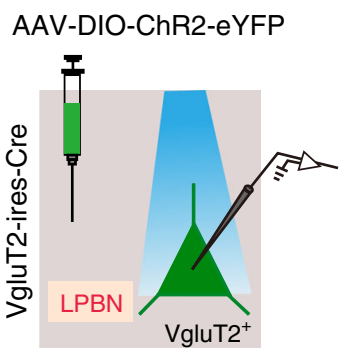

b

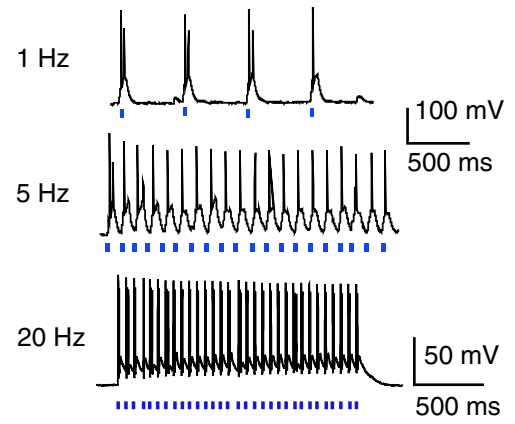

C

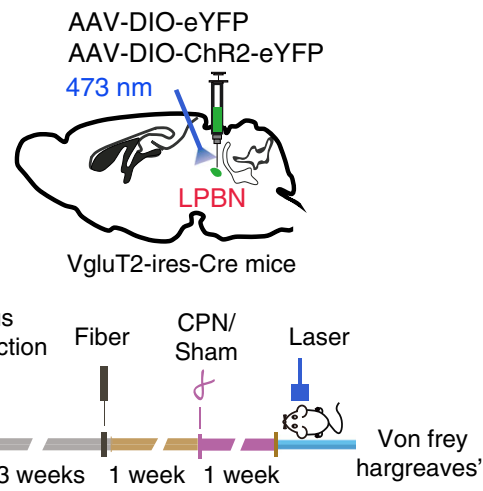

d

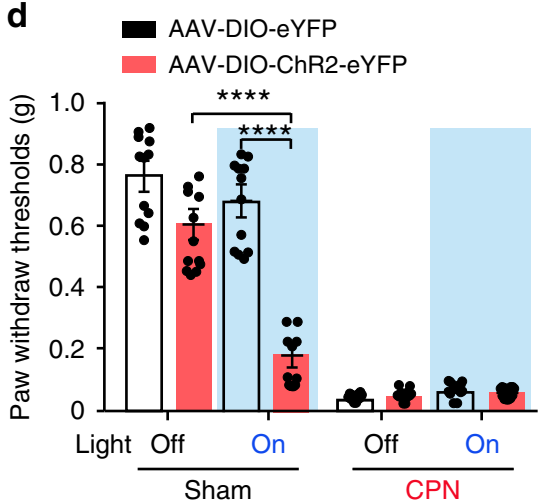

e

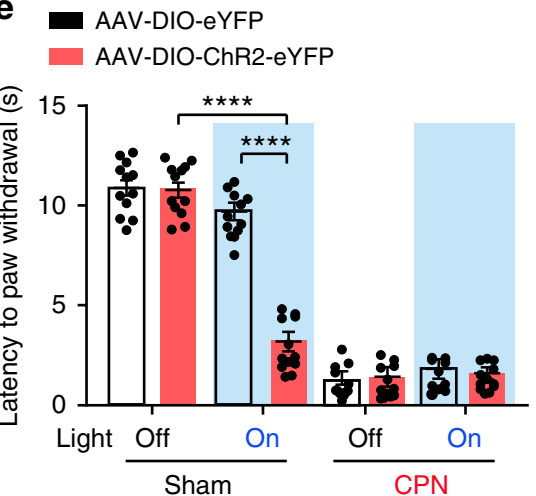

f

Real-time place avoidance (RTPA)

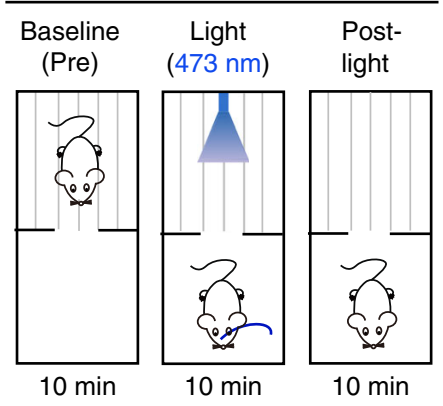

g

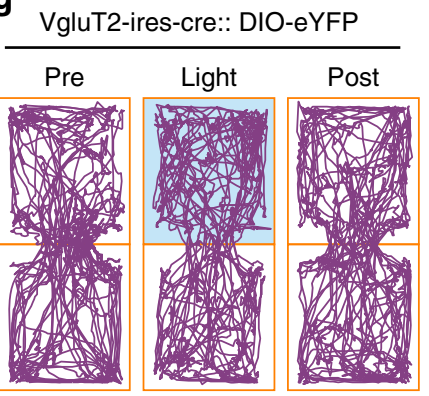

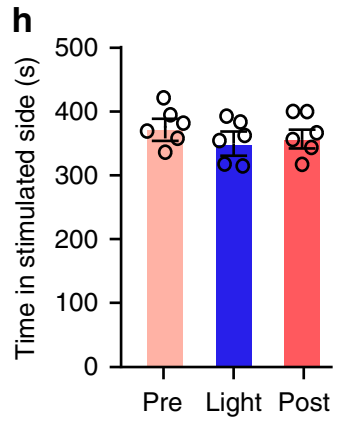

i

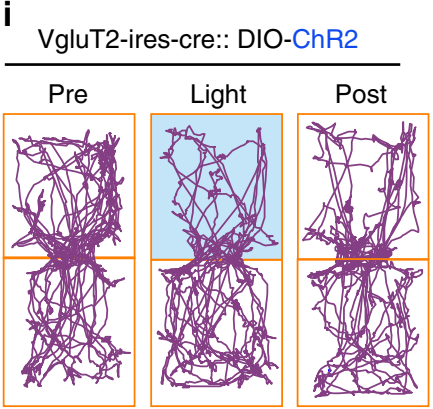

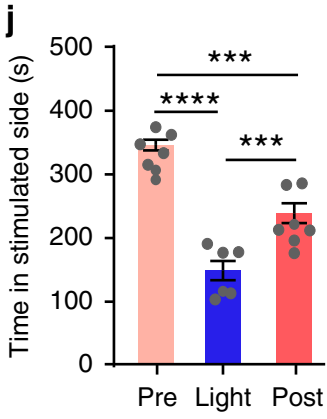

Fig. 2 Optogenetic activation of VgluT2 neurons in LPBN induces hyperalgesia and place avoidance behavior. a Schematic of light stimulation of and patch-clamp recording from glutamatergic LPBN neurons transfected with AAV-Cre-on-ChR2-eYFP in brain slices from VgluT2-ires-Cre mice. $\mathbf{b}$ Example patch-clamp recordings showing light stimulation-induced time-locked action potential firing in ChR2-expressing VgluT2 neurons in the LPBN. Blue bars indicate application of light stimuli ( $473 \mathrm{~nm}, 5 \mathrm{~ms}, \sim 5 \mathrm{~mW}$ ) at $1 \mathrm{~Hz}$ (upper), $5 \mathrm{~Hz}$ (middle), and $20 \mathrm{~Hz}$ (lower). c Schematic of stereotaxic delivery of AAV carrying Cre-dependent ChR2 into the LPBN of VgluT2-ires-Cre mice (upper) and experimental design and timeline of the behavioral experiments (lower). d Illumination ( $473 \mathrm{~nm}, 20 \mathrm{~Hz}$, 5-ms pulse width, $5 \mathrm{~mW}$ ) of the LPBN significantly decreases the paw-withdraw thresholds (PWT) in response to von Frey mechanical stimulation in Sham-operated mice transfected with ChR2-eYFP, but not in mice transfected with eYFP in VgluT2 LPBN neurons. e Illumination $(473 \mathrm{~nm}, 20 \mathrm{~Hz}, 5$-ms pulse width, $\sim 5 \mathrm{~mW}$ ) of the LPBN significantly decreases the latency of the thermal paw-withdraw response in the Hargreaves test in Sham-operated mice transfected with ChR2-eYFP, but not in mice transfected with eYFP in VgluT2 LPBN neurons. f Schematic of the real-time place avoidance (RTPA) test. $\mathbf{g}-\mathbf{j}$ Representative tracks $(\mathbf{g}, \mathbf{i})$ and quantification of time spent in the preferred chamber ( $\mathbf{h}, \mathbf{j})$ in the RTPA test before (Pre), during (Light), and immediately after (Post) laser illumination ( $473 \mathrm{~nm}, 20 \mathrm{~Hz}, 5$-ms pulse width, $\sim 5 \mathrm{~mW}$ ) of the LPBN transfected with eYFP ( $\mathbf{g}$, $\mathbf{h}$ ) or ChR2 (i, $\mathbf{j}$ ) in Vgltu2-ires-Cre mice. All data are presented as mean \pm s.e.m. and error bars represent s.e.m. ${ }^{\star \star \star} P<0.001$ and ${ }^{\star \star \star \star} P<0.0001$. See also Supplementary Table 1 for further statistical information. Source data are provided as a Source Data file.

found that photoinhibition of LPBNCaMKIIa or LPBNVgluT2 neurons did not affect the total distance moved and the ratio of time spent in the periphery and center of the open field in Sham-operated or CPN-ligated mice, suggesting that the locomotor and anxiety levels were not altered by optogenetic manipulation (Figs. 4h-m, 5g-k). Taken together, these data demonstrate that silencing LPBNCaMKIIa or LPBNVgluT2 neurons not only alleviates basal nociception but also relieves both the sensory and affective components of neuropathic pain-like hypersensitivity.
Activation of GABAergic LPBN neurons alleviates neuropathic pain-like hypersensitivity but not basal nociception. The results that GABAergic neurons constitute only $10.1 \%$ of LPBN neurons and that most of the neurons activated in the LPBN after CPN ligation are glutamatergic (Fig. 1a-h) prompted us to investigate whether the activation of local GABAergic neurons has any effect on basal nociception and neuropathic pain-like hypersensitivity. To address this issue, optical fibers were implanted bilaterally into the LPBN of transgenic mice with vesicular GABA transporter (vGAT)-ChR2 (H134R)-eYFP (vGAT-ChR2). The activation of 
a

AAV-CaMKII $\alpha-m$ Cherry AAV-CaMKII $\alpha$-ChR2-mCherry

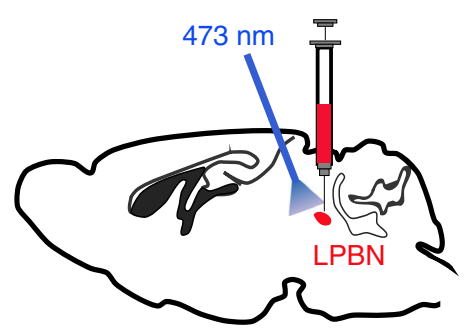

Wild-type mice b

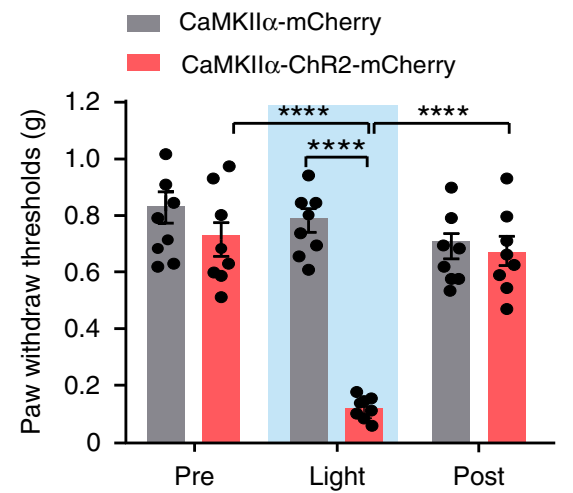

C

$$
\begin{aligned}
& \text { CaMKIl } \alpha-m \text { Cherry } \\
& \text { CaMKIl } \alpha \text {-ChR2-mCherry }
\end{aligned}
$$

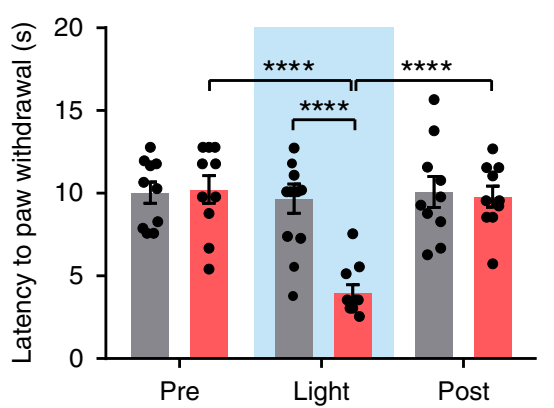

d

CaMKIl $\alpha$-mCherry: RTPA

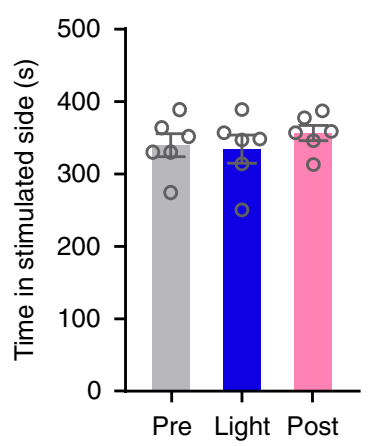

e

\section{CaMKIl $\alpha-C h R 2:$ RTPA}

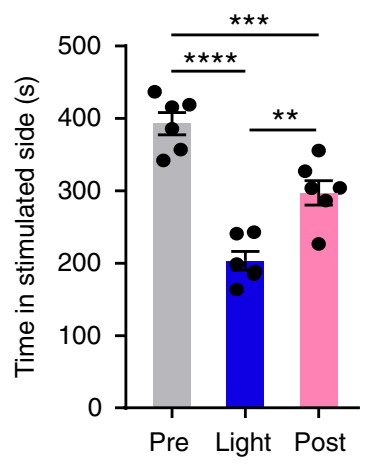

$\mathbf{f}$

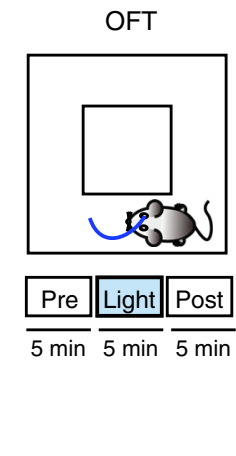

g

CaMKIILmCherry

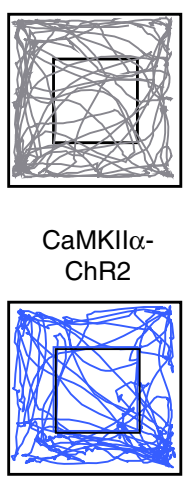

h

h $\square$ CaMKIl $\alpha-m$ Cherry CaMKIl $\alpha$-ChR2-mCherry

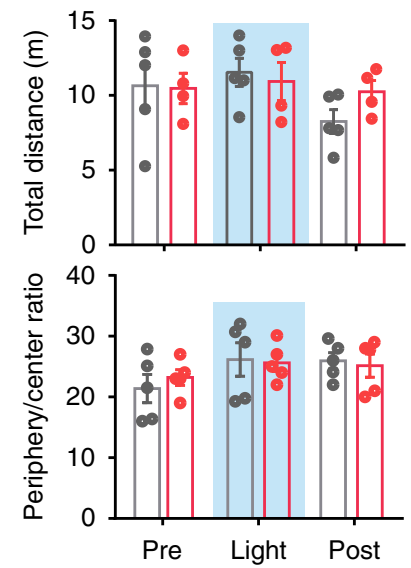

Fig. 3 Optogenetic activation of LPBNCaMKIl $\alpha$ neurons induces hyperalgesia and place avoidance behavior. a Schematic of the stereotaxic delivery of AAV carrying CaMKIl $\alpha$-ChR2 into the LPBN of wild-type mice. $\mathbf{b}$ Light activation ( $473 \mathrm{~nm}, 20 \mathrm{~Hz}, 5$-ms pulse width, $\sim 5 \mathrm{~mW}$ ) of LPBNCaMKIll neurons significantly decreases the PWT induced by von Frey stimulation in mice injected with AAV-CaMKIl $\alpha$-ChR2-mCherry, compared with mice injected with AAV-CaMKIl $\alpha$-mCherry. No significant difference was found between before (Pre) and after (Post) light stimulation. $\mathbf{c}$ Light activation (473 nm, $20 \mathrm{~Hz}$, 5ms pulse width, $\sim 5 \mathrm{~mW}$ ) of LPBNCaMKIlo neurons significantly decreases thermal paw-withdrawal latency measured by the Hargreaves test in mice injected with AAV-CaMKIl $\alpha$-ChR2-mCherry, compared with mice injected with AAV-CaMKIl $\alpha$-mCherry. No significant difference was found between before (Pre) and after (Post) light stimulation. d, e Quantification of time spent in the preferred chamber in the RTPA test before (Pre), during (Light), and after (Post) 10-min illumination of the LPBN in mice transfected with AAV-CaMKIl $\alpha$-mCherry (d) and AAV-CaMKIl $\alpha$-ChR2-mCherry (e). f Schematic of the open field test (OFT) with photostimulation via a 473-nm laser (Pre, Light, and Post; 5 min for each stage). $\mathbf{g}$ Example traces of the OFT from mice with AAVCaMKIl $\alpha$-mCherry (upper) and AAV-CaMKIl $\alpha$-ChR2-mChreey (lower) injected into the LPBN. $\mathbf{h}$ Quantification of the total distance moved (upper) and ratio of time spent in the periphery and center (lower) in the OFT before (Pre), during (Light), and after (Post) 10-min illumination of the LPBN in wild-type mice transfected with AAV-CaMKIl $\alpha-m C h e r r y$ and AAV-CaMKIl $\alpha$-ChR2-mCherry. All data are presented as mean \pm s.e.m. and error bars represent s.e.m. ${ }^{\star \star \star} P<0.001$ and ${ }^{\star \star \star \star} P<0.0001$. See also Supplementary Table 1 for further statistical information. Source data are provided as a Source Data file.

GABAergic neurons by photostimulation in the LPBN was confirmed by double-labeling for vGAT and c-Fos (Fig. 6a). Surprisingly, we found that although optogenetic activation of GABAergic LPBN neurons failed to affect the basal nociception in Sham-operated mice, it induced a significant, time-locked increase in the threshold and latency of the paw-withdrawal responses evoked by mechanical and thermal stimulation in CPN-ligated mice to levels comparable to those in Sham-operated mice (Fig. 6b, c). Furthermore, light stimulation of the GABAergic LPBN neurons significantly increased the time spent in the chamber paired with photostimulation in CPN-ligated mice, but not in controls, in the RTPP test (Fig. 6d-g), consistent with the light stimulation-induced pain relief in CPN-ligated mice.

Since the LPBN also receives GABAergic innervation from other brain regions ${ }^{28,53,54}$, it was possible that light stimulation in the vGAT-ChR2 transgenic mice activated the fibers and terminals of GABAergic neurons projecting to or via the LPBN from other brain regions. To determine whether activation of the local GABAergic LPBN neurons is sufficient to inhibit neuropathic pain-like hypersensitivity, we initially injected Credependent AAV-DIO-ChR2-eYFP or AAV-DIO-eYFP virus into the LPBN of mice expressing Cre recombinase under the control of the glutamic acid decarboxylase enzyme (GAD2-ires-Cre mice) (Fig. 6h and Supplementary Fig. 4). A dramatic increase in the threshold and latency of the paw-withdrawal responses induced by mechanical and thermal stimulation was found in CPN-ligated mice during illumination of LPBN neurons transfected with AAV-DIO-ChR2-eYFP in GAD2-ires-Cre mice, but neither in CPN-ligated mice transfected with AAV-DIO-eYFP nor in Shamoperated mice (Fig. 6i, j). Similarly, the time spent in the chamber paired with photostimulation was significantly increased in CPNligated mice in the RTPP test during and $10 \mathrm{~min}$ after light stimulation of the LPBN transfected with AAV-DIO-ChR2-eYFP, 
a

AAV-CaMKII $\alpha$-mCherry AAV-CaMKIl $\alpha-e N p H R-m C h e r r y$

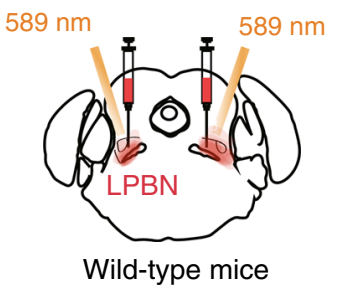

b
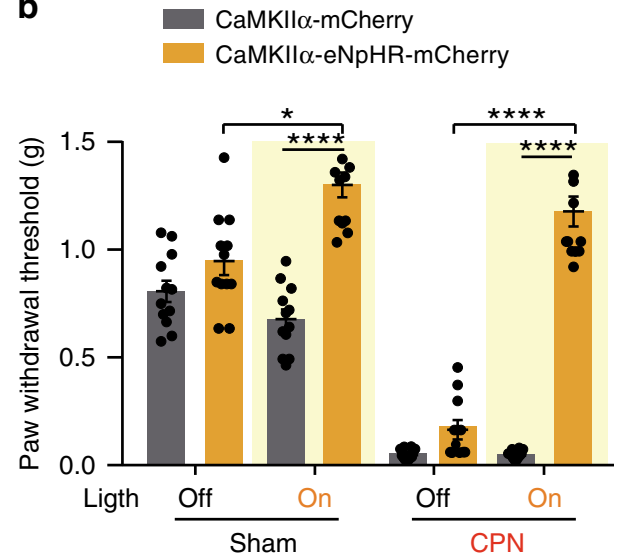

h

d

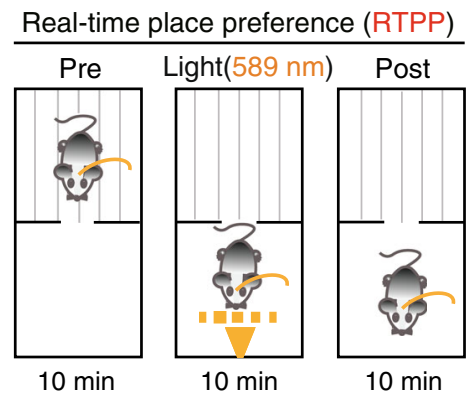

e
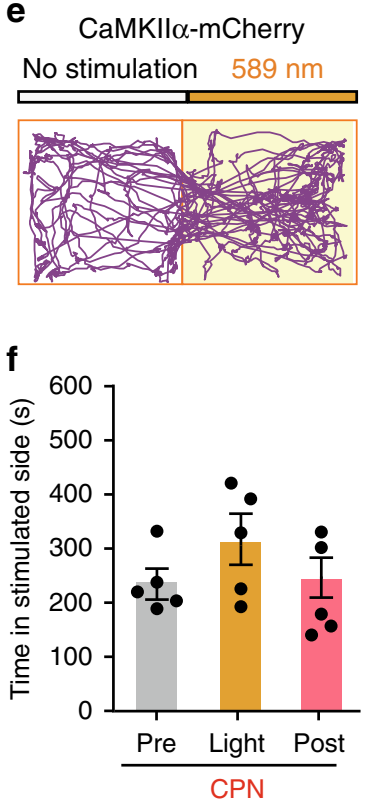

CaMKII $\alpha-e N p H R$
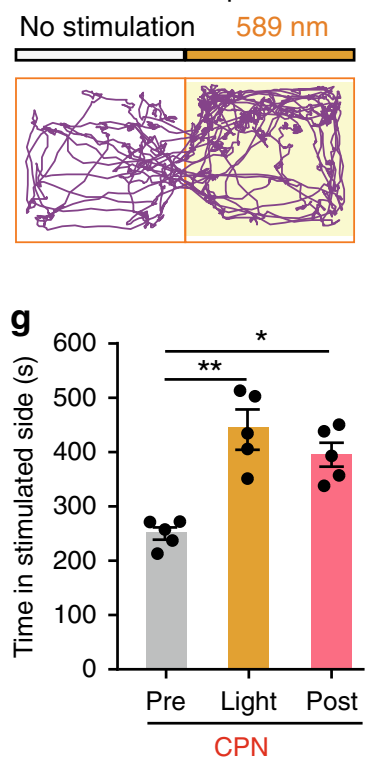

j

j

$\mathbf{k}$

but not with AAV-DIO-eYFP (Fig. 6k). Optogenetic activation of GABAergic LPBN neurons in CPN-ligated mice did not affect the total distance moved and the ratio of time spent in the periphery and center of the open field (Fig. 6l), suggesting that the locomotor and anxiety levels were not altered by optogenetic manipulation. These results demonstrated that activation of local GABAergic LPBN neurons, while leaving the basal nociception unaffected, efficiently alleviates neuropathic pain-like hypersensitivity in terms of both sensory discrimination and emotional behavior.

We next used the pharmacogenetic approach to further confirm the effect of activating GABAergic LPBN neurons on neuropathic pain-like hypersensitivity. We bilaterally injected the

C
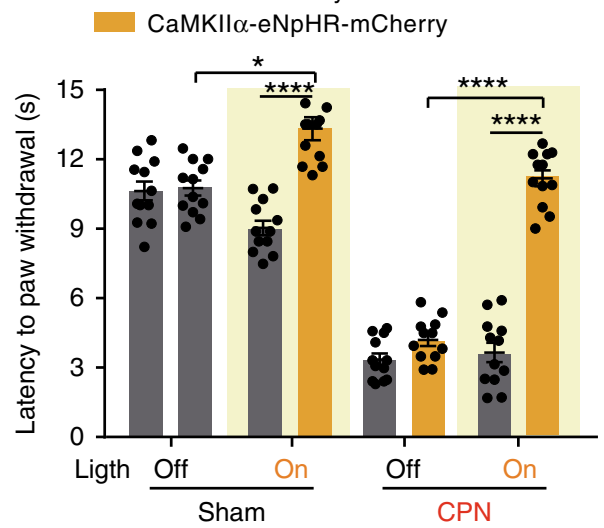

i

CaMKII $\alpha-m$ Cherry $\quad$ CaMKIl $\alpha-e N p H R$
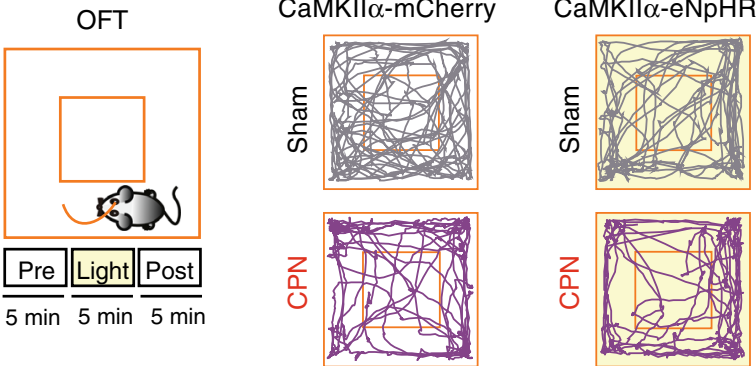

I
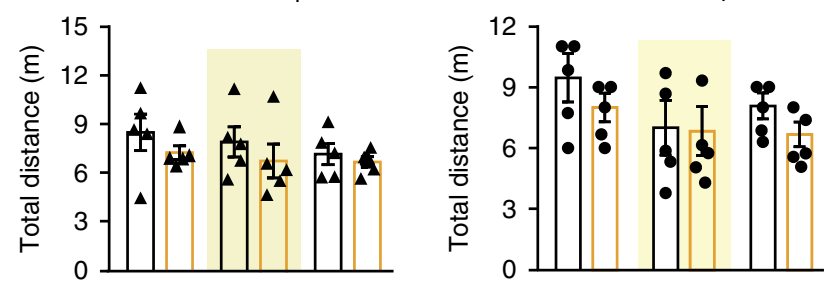

m
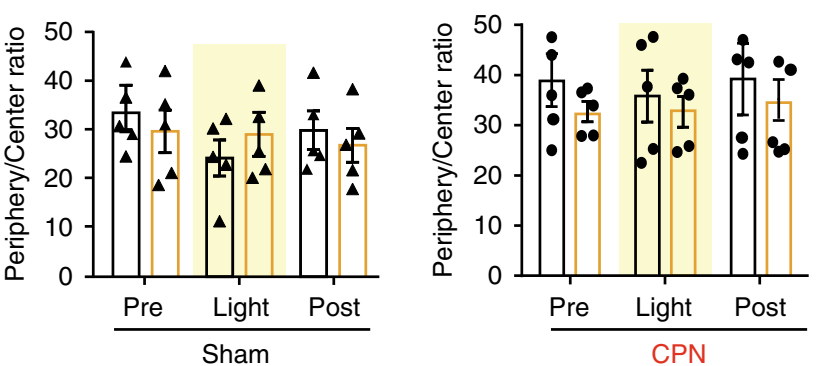

LPBN of GAD2-ires-Cre mice with an AAV expressing hM3Dq, a designer receptor exclusively activated by designer drugs $(\mathrm{DREADD})^{55}$ or mCherry as control (Fig. 6m). Three weeks after virus infection, CPN ligation was carried out, and a week later, the hM3Dq agonist clozapine-N-oxide (CNO) was injected intraperitoneally (i.p.) once per day for 4 days (Fig. 6m). As expected, the decreased PWT induced by CPN ligation was elevated on the first day of $\mathrm{CNO}$ injection and was further elevated after the subsequent daily injections in mice expressing hM3Dq in the GABAergic LPBN neurons, but not in mice transfected with mCherry (Fig. 6n). Interestingly, delivery of picrotoxin (PTX, a blocker of the $\mathrm{GABA}_{\mathrm{A}}$ receptor chloride channel) into the LPBN via an intracerebral cannula completely 


\begin{abstract}
Fig. 4 Photoinhibition of LPBNCaMKIllo neurons alleviates both basal nociception and neuropathic pain-like hypersensitivity. a Schematic of the light illumination and virus injection strategy for the expression of CaMKIl $\alpha$-eNpHR or CaMKIl $\alpha$-mCherry in the LPBN of wild-type mice. b Yellow illumination $(589 \mathrm{~nm}, 1 \mathrm{~Hz}, 999-\mathrm{ms}$ pulse width, $10 \mathrm{~mW}$ ) of the LPBN significantly elevates the PWT in response to mechanical stimulation in both Sham-operated and CPN-ligated mice transfected with AAV-CaMKIl $\alpha$-eNpHR, but not in mice transfected with CaMKIl $\alpha$-mCherry in the LPBN. c Yellow illumination (589 $\mathrm{nm}, 1 \mathrm{~Hz}$, 999-ms pulse width, $10 \mathrm{~mW}$ ) of the LPBN significantly increases the latency of the thermal paw-withdrawal response in the Hargreaves test in both Sham-operated and CPN-ligated mice transfected with AAV-CaMKIl $\alpha$-eNpHR, but not in mice transfected with CaMKIl $\alpha$-mCherry in the LPBN. d Schematic of the real-time place preference (RTPP) test. e Representative tracks of RTPP illustrating yellow (589 nm) light-evoked behavioral preference in CPN-ligated mice injected with AAV-CaMKIl $\alpha$-mCherry (left) or CaMKIl $\alpha$-eNpHR (right) virus in the LPBN. f, $\mathbf{g}$ Quantification of RTPP in CPN-ligated mice before (Pre), during (Light), and after (Post) 10-min yellow illumination of the LPBN in mice injected with AAV-CaMKIl $\alpha$-mCherry (f) or CaMKIl $\alpha$ eNpHR (g). h Schematic of the open field test (OFT) before (Pre), during (Light), and after (Post) 5 min photoinhibition ( $589 \mathrm{~nm}, 1 \mathrm{~Hz}, 999-\mathrm{ms}$ pulse width, $10 \mathrm{~mW}$ ) of LPBNCaMKIl $\alpha$ neurons. i Representative tracks of mice transfected with CaMKIl $\alpha$-mCherry (left) or CaMKIl $\alpha$-eNpHR (right) in the LPBN with yellow laser illumination $(589 \mathrm{~nm}$ ) in Sham-operated (upper) or CPN-ligated (lower) wild-type mice. j-m Quantification of total distance moved (j, I) and ratio of time spent in the periphery and center $(\mathbf{k}, \mathbf{m})$ in the OFT before (Pre), during (Light), and after (Post) 5 min yellow light illumination of the LPBN in mice transfected with CaMKIl $\alpha$-mCherry or CaMKIl $\alpha$-eNpHR in Sham-operated $(\mathbf{j}, \mathbf{k})$ and CPN-ligated (I, $\mathbf{m})$ mice. All data are presented as mean \pm s.e.m. and error bars represent s.e.m. ${ }^{\star} P<0.05,{ }^{\star \star} P<0.01$, and ${ }^{\star \star \star \star}{ }^{\star} P<0.0001$. See also Supplementary Table 1 for further statistical information. Source data are provided as a Source Data file.
\end{abstract}

reversed the effect of $\mathrm{CNO}$ (Fig. 6n), indicating that GABAergic neurons control neuropathic pain-like hypersensitivity through locally-released GABA in the LPBN, rather than through their projections to other regions outside the LPBN. We found that pharmacogenetic activation of GABAergic LPBN neurons by $\mathrm{CNO}$ in $\mathrm{CPN}$-operated mice also induced a conditioned place preference (CPP) in the chamber paired with i.p. injection of CNO (Supplementary Fig. 5a-c), indicating the relief of the aversive emotion accompanying neuropathic pain-like hypersensitivity. Activation of GABAergic LPBN neurons by $\mathrm{CNO}$ treatment was confirmed by immunostaining of c-Fos expression in the LPBN (Supplementary Fig. 5d, e).

Inactivation of GABAergic LPBN neurons is sufficient to induce hyperalgesia and the place avoidance response. The above results-that activation of GABAergic LPBN neurons alleviates neuropathic pain-like hypersensitivity through local inhibition of glutamatergic neuronal activity and that activation of glutamatergic LPBN neurons is sufficient to induce hyperalgesia mimicking neuropathic pain-prompted us to investigate whether the inactivation of GABAergic LPBN neurons affects pain processing. To do this, AAVs carrying Cre-dependent GtACR1 (Guillardia theta anion channel rhodopsins 1$)^{56}$ was injected into the LPBN of GAD2-ires-Cre mice (Fig. 7a). In contrast to the activation of GABAergic LPBN neurons, which did not affect the basic pain threshold in Sham-operated mice (Fig. 6b, c), we found that light inactivation of GABAergic LPBN neurons drastically decreased the threshold and latency of the paw-withdrawal responses evoked by mechanical allodynia (Fig. 7b) and thermal stimulation (Fig. 7c) in naïve (without CPN ligation) GAD2-ires-Cre mice transfected with GtACR1-eYFP to a level comparable to that in $\mathrm{CPN}$-ligated mice, while illumination of GABAergic LPBN neurons transfected with eYFP had no effect (Fig. 7b, c). Furthermore, illumination of GABAergic LPBN neurons transfected with GtACR1-eYFP, but not with eYFP, significantly decreased the time spent in the chamber paired with illumination in the RTPA test (Fig. 7d, e). Optogenetic inactivation of GABAergic LPBN neurons did not affect the total distance moved and the ratio of time spent in the periphery and center in the open field test (Fig. 7f), indicating that the motor performance and baseline anxiety level were not changed by optogenetic manipulation.

Consistent with this, we found that light inactivation of GABAergic LPBN neurons transfected with GtACR1-eYFP induced a robust increase $(\sim 4.0$-fold $)$ in c-Fos expression in glutamatergic $\left(\mathrm{CaMKIIa}^{+}\right)$but not in GABAergic LPBN neurons, compared with mice transfected with eYFP (Fig. $7 \mathrm{~g}-\mathrm{i}$ ). Similar to the CPN-ligation-induced c-Fos expression (Fig. 1a-d), $~ 80.1 \%$ of the c-Fos-positive neurons overlapped with CaMKIIa, while only $\sim 2.6 \%$ of the c-Fos-positive neurons overlapped with eYFP (GABAergic neurons) (Fig. 7j). These results demonstrate that, similar to optogenetic activation of the glutamatergic LPBN neurons (Fig. 2d, e), optogenetic inactivation of the GABAergic LPBN neurons also mimics the typical symptoms of neuropathic pain, suggesting that tonic activity of GABAergic neurons is critical for preventing neuropathic pain-like hypersensitivity.

Monosynaptic innervation of glutamatergic LPBN neurons by local GABAergic neurons. The above results suggest that GABAergic LPBN neurons modulate neuropathic pain-like hypersensitivity by directly inhibiting glutamatergic LPBN neurons. To address this possibility, we first used a monosynaptic retrograde tracing viral tool by injecting Cre-dependent expression helper virus including AAV-CAG-DIO-TVA-eGFP and AAV-CAG-DIO-RG on day 1 and RV-EnvA-DsRed on day 21 into the LPBN of VgluT2-ires-Cre mice (Fig. 8a). We found that some trans-synaptically infected LPBN neurons expressing DsRed were GABAergic neurons as identified by in situ hybridization for GAD1 mRNA expression (Fig. 8b), suggesting that glutamatergic LPBN neurons receive direct monosynaptic innervation from local GABAergic neurons.

To further electrophysiologically demonstrate the direct synaptic innervation of LPBNCaMKIIa neurons by local GABAergic interneurons, we simultaneously injected two types of virus, AAV-CaMKIIa-mCherry and AAV-DIO-ChR2-eYFP, into the LPBN of GAD2-ires-Cre mice (Fig. 8c, d). Voltage-clamp recordings from LPBNCaMKIIa neurons expressing mCherry in brain slices showed that light stimulation of GABAergic neurons expressing ChR2-eYFP optically evoked inhibitory postsynaptic currents (eIPSCs), which were evident as an inward current when neurons were recorded with a high $\mathrm{Cl}^{-}$intra-pipette solution (135 mM), at a holding membrane potential of $-70 \mathrm{mV}$ (Fig. 8e). Several lines of evidence indicated that this light-evoked synaptic current resulted from the direct activation of monosynapticallyconnected GABAergic neurons. That is, the light-evoked synaptic current had a short latency $(4.4 \mathrm{~ms}$, Fig. $8 \mathrm{~g})$, and these eIPSCs were sensitive to the $\mathrm{Na}^{+}$channel blocker tetrodotoxin (TTX, 1 $\mu \mathrm{M})$, which abolished action potentials propagating to the synaptic terminals so that the synaptic transmission induced by light-evoked action potentials was blocked. However, in the presence of the $\mathrm{K}^{+}$channel blocker 4-aminopyridine (4-AP, 100 $\mu \mathrm{M})$, which reduces membrane leakage current and thus enables the direct depolarization of synaptic terminals expressing ChR2 by light stimulation ${ }^{18,19}$, TTX failed to block the light-evoked synaptic current in LPBNCaMKIIa neurons (Fig. 8e). These findings, together with the result that the TTX-insensitive 
a

AAV-DIO-eYFP

AAV-DIO-eNpHR3.0-eYFP

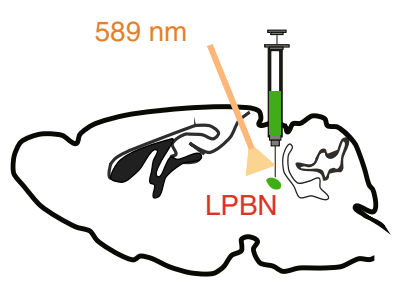

VgluT2-ires-cre mice b

O AAV-DIO-eYFP

O AAV-DIO-eNpHR-eYFP

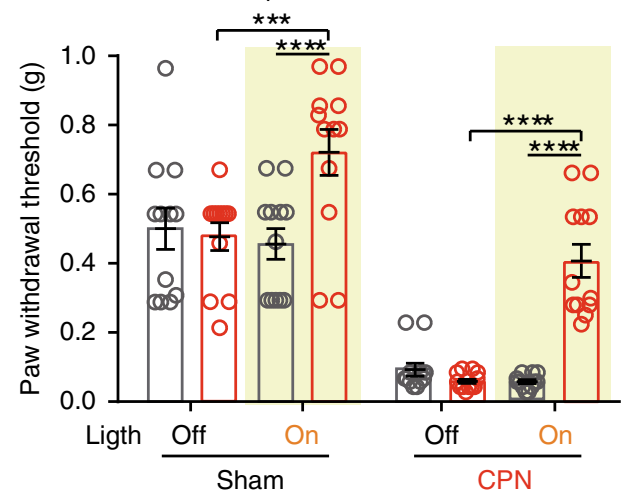

C

O AAV-DIO-eYFP

O AAV-DIO-eNpHR-eYFP

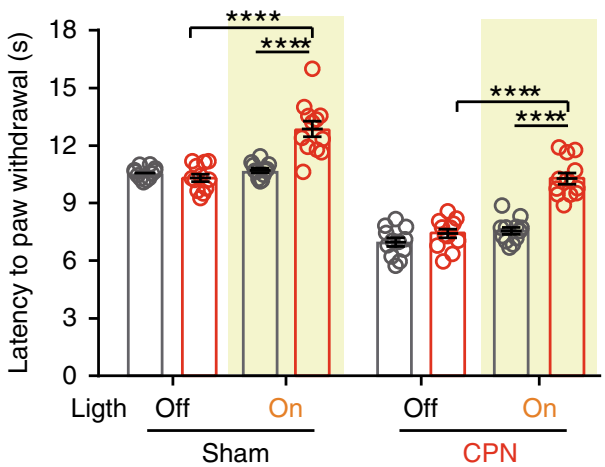

d

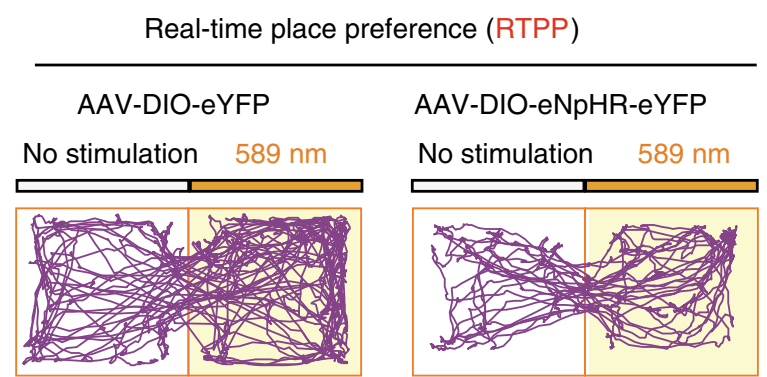

g

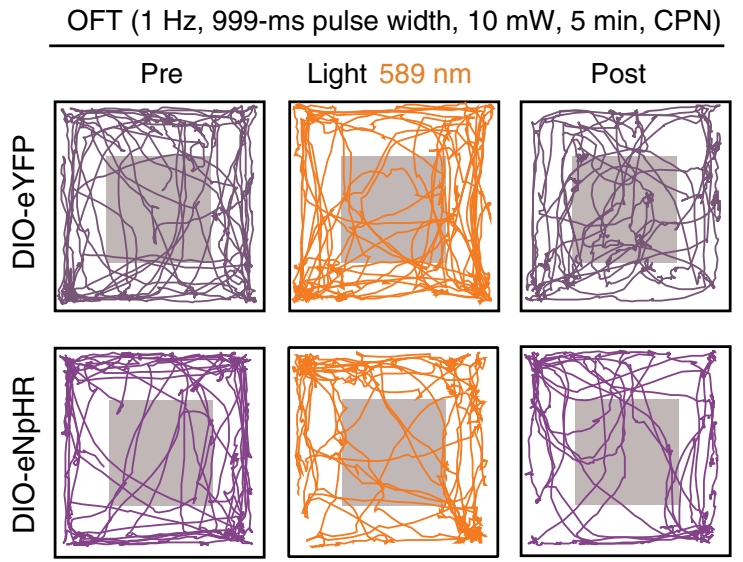

e

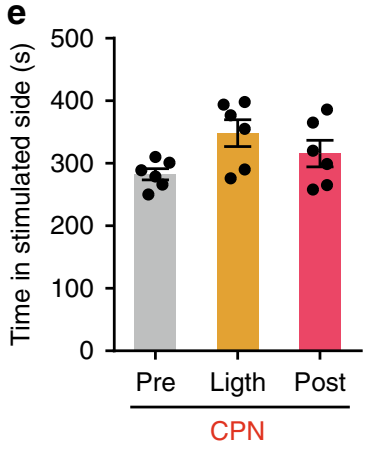

h
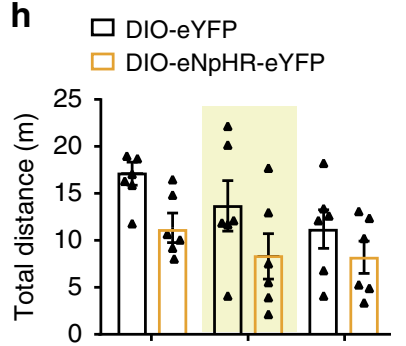

i

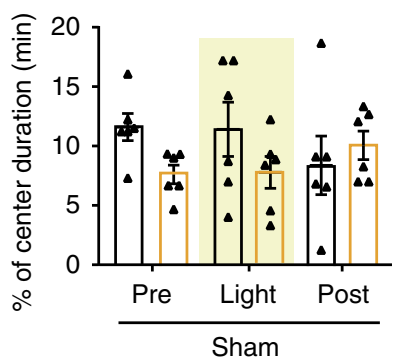

f

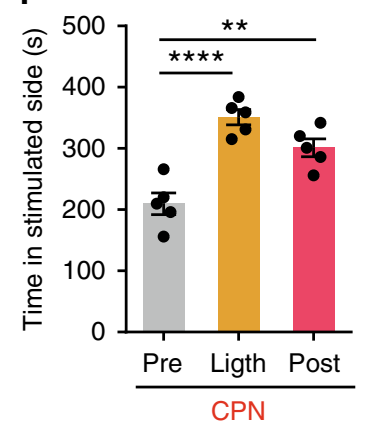

j $\square$ DIO-eYFP $\square$ DIO-eNpHR-eYFP

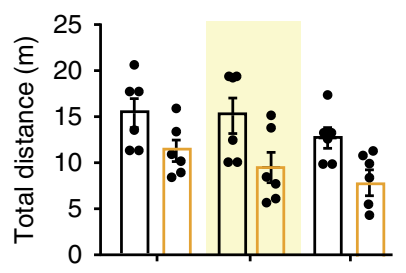

k

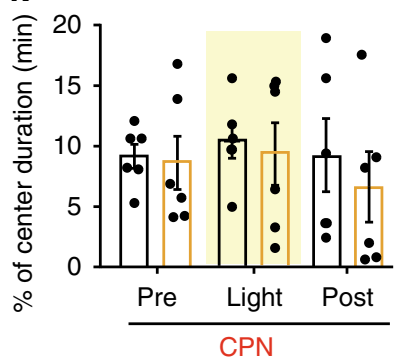

component was blocked by the $\mathrm{GABA}_{\mathrm{A}}$ receptor antagonist bicuculline $(10 \mu \mathrm{M})$ (Fig. $8 \mathrm{e})$, further confirmed the monosynaptic innervation of LPBNCaMKIIa neurons by local GABAergic neurons.

To determine whether activation of GABAergic LPBN neurons functionally modulates glutamatergic LPBN neurons, LPBNCaM$\mathrm{KII} \alpha$ neurons expressing mCherry in brain slices were recorded with a lower intra-pipette $\mathrm{Cl}^{-}$concentration $(6 \mathrm{mM})$ under current-clamp with a membrane potential held around $-45 \mathrm{mV}$ so that spontaneous spikes could be detected (Supplementary Fig. 6c). We found that brief light stimulation (1 ms pulses at 20
$\mathrm{Hz}$ for $5 \mathrm{~s}$ ) of GABAergic neurons expressing ChR2 significantly inhibited the spike frequency of LPBNCaMKIla neurons for $>10 \mathrm{~s}$, accompanied by membrane hyperpolarization (Fig. 8h-k), indicating inhibited activity of LPBNCaMKIIa neurons mediated by local GABAergic neurons. We also found that CPN treatment increased the paired-pulse ratio of IPSCs induced by light stimulation of GABAergic LPBN neurons (Supplementary Fig. 6d, e), suggesting a decreased release probability of GABAergic synaptic vesicles ${ }^{57}$. On the other hand, the density of GABAergic LPBN neurons was not affected by CPN treatment (Supplementary Fig. 6a, b). 
Fig. 5 Photoinhibition of LPBNVgluT2 neurons alleviates both basal nociception and neuropathic pain-like hypersensitivity. a Schematic of illumination and virus injection of AAV-DIO-eYFP and AAV-DIO-eNpHR-eYFP in the LPBN of VgluT2-ires-Cre mice. b Yellow illumination ( $589 \mathrm{~nm}, 1 \mathrm{~Hz}, 999-\mathrm{ms}$ pulse width, $10 \mathrm{~mW}$ ) of LPBNVglut2 neurons significantly elevates the PWT in response to mechanical stimulation in both Sham-operated and CPN-ligated VgluT2ires-Cre mice transfected with AAV-DIO-eNpHR virus, but not in mice transfected with DIO-eYFP virus. c Yellow illumination ( $589 \mathrm{~nm}, 1 \mathrm{~Hz}, 999-\mathrm{ms}$ pulse width, $10 \mathrm{~mW}$ ) of the LPBN significantly increases the latency of the thermal paw-withdrawal response in the Hargreaves test in both Sham-operated and CPN-ligated VgluT2-ires-Cre mice transfected with AAV-DIO-eNpHR virus, but not in mice transfected with DIO-eYFP virus. d Representative tracks of RTPP illustrating yellow $(589 \mathrm{~nm}$ ) light-evoked behavioral preference in CPN-ligated VgluT2-ires-Cre mice injected with AAV-DIO-eYFP (left) or DIOeNpHR (right) virus in the LPBN. e, f Quantification of RTPP in CPN-ligated mice before (Pre), during (Light), and after (Post) 10-min yellow illumination of the LPBN in VgluT2-ires-Cre mice injected with AAV-DIO-eYFP (e) or AAV-DIO-eNpHR (f) virus. $\mathbf{g}$ Representative tracks in the open field test (OFT) before (Pre), during (Light), and after (Post) 5-min yellow laser illumination ( $589 \mathrm{~nm}, 1 \mathrm{~Hz}, 999$-ms pulse width, $10 \mathrm{~mW}$ ) of LPBNVglut2 neurons transfected with DIO-eYFP (upper) or DIO-eNpHR (lower) virus in CPN-ligated VgluT2-ires-Cre mice. $\mathbf{h}$-k Quantification of total distance moved (h, $\mathbf{j}$ ) and ratio of time spent in the periphery and center $(\mathbf{i}, \mathbf{k})$ in the OFT in VgluT2-ires-Cre mice transfected with DIO-eYFP or DIO-eNpHR virus in Sham-operated ( $(\mathbf{h}, \mathbf{i})$ and CPN-ligated (j, k) mice. All data are presented as mean \pm s.e.m. and error bars represent s.e.m. ${ }^{\star} P<0.05,{ }^{\star \star} P<0.01$, and ${ }^{\star \star \star \star} P<0.0001$. See also Supplementary Table 1 for further statistical information. Source data are provided as a Source Data file.

Prolonged activation of glutamatergic LPBN neurons induces chronic hyperalgesia. It has been suggested that neuropathic pain results from plastic changes in the structure and function of the pain processing pathway induced by prolonged nociceptive signaling ${ }^{1,6}$. Since the photoactivation of glutamatergic LPBN neurons instantly induced hyperalgesia, we next investigated whether prolonged activation of these neurons can induce persistent pain, mimicking the development of neuropathic pain. To do this, we induced repetitive pharmacogenetic activation of glutamatergic LPBN neurons by daily intraperitoneal injection of CNO $\left(1.5 \mathrm{mg} \mathrm{kg}^{-1}\right)$ for a week in VgluT2-ires-Cre mice with bilateral injection of AAV-DIO-hM3Dq-mCherry (hM3Dq) or AAV-DIO-mCherry (mCherry) into the LPBN (Fig. 9a). We found that daily $\mathrm{CNO}$ injection for one week induced a persistent decrease in the PWT for more than one month in mice expressing hM3Dq but not in those expressing mCherry (Fig. 9b), indicating that prolonged activation of glutamatergic LPBN neurons is sufficient to induce the development of chronic pain-like hypersensitivity.

We further determined whether this prolonged pharmacogenetic activation of glutamatergic LPBN neurons is sufficient to induce an aversive affective memory using the conventional conditioned place aversion (CPA) behavioral paradigm. Mice with hM3Dq or mCherry expression in glutamatergic LPBN neurons were habituated to two communicating chambers for 30 $\min$ on days 1 and 2 . On day 3 , the mice were tested for $10 \mathrm{~min}$ to confirm that there was no preference for one of the chambers. From day 4, the mice were injected (i.p.) with saline and paired to one side of the chamber for $30 \mathrm{~min}$, and $4 \mathrm{~h}$ later, the mice were injected with $\mathrm{CNO}$ and paired to the other side for $30 \mathrm{~min}$. The saline and $\mathrm{CNO}$ were injected once per day for 3 days. Twentyfour hours after the last injection of $\mathrm{CNO}$, the mice were free to move to both sides, and the time in each chamber was measured (Fig. 9c). We found that the CNO injection induced significant place aversion for the $\mathrm{CNO}$-paired chamber in mice transfected with hM3Dq, but not in those transfected with mCherry in the LPBN (Fig. 9d, e).

To determine whether this pharmacogenetic-induced chronic hyperalgesia can also be alleviated by the activation of GABAergic LPBN neurons, we simultaneously injected two types of virus into the LPBN of GAD2-ires Cre mice: AAVCaMKIIa-hM3Dq-mCherry for the repetitive pharmacogenetic activation of glutamatergic LPBN neurons by daily $\mathrm{CNO}$ injections for a week, and Cre-dependent AAV-DIO-ChR2eYFP for the instant optogenetic activation of GABAergic neurons (Fig. 9f). We found that the optogenetic activation of GABAergic LPBN neurons transfected with DIO-ChR2 transiently reversed the mechanical allodynia induced by $\mathrm{CNO}$ injection in mice transfected with CaMKIIa-hM3Dq in glutamatergic LPBN neurons (Fig. 9g, red line, and day 21). Taken together, these results indicate that prolonged activation of glutamatergic LPBN neurons alone is sufficient to induce fully-developed chronic hyperalgesia that mimics CPN ligationinduced neuropathic pain-like hypersensitivity.

Prolonged activation of GABAergic LPBN neurons prevents the development of neuropathic pain-like hypersensitivity. The results that $\mathrm{CPN}$ ligation activated glutamatergic but not GABAergic LPBN neurons and that the prolonged selective pharmacogenetic activation of glutamatergic LPBN neurons was sufficient to induce chronic pain suggested that an imbalance between the excitatory and inhibitory activity of neurons in the LPBN may be responsible for the development of neuropathic pain-like hypersensitivity. Since local GABAergic neurons monosynaptically control glutamatergic LPNB neuronal activity and the activation of GABAergic LPBN neurons instantly alleviates neuropathic pain-like hypersensitivity, we next investigated whether prolonged activation of GABAergic LPBN neurons during the early stage (first week) of CPN ligation affects the development of neuropathic pain-like hypersensitivity. To do this, AAV-DIO-hM3Dq-mCherry or AAV-DIO-mCherry was bilaterally injected into the LPBN of GAD2-ires-Cre mice. Three weeks after virus infection, the mice received $\mathrm{CPN}$ ligation surgery, followed by daily CNO injections (i.p) for a week (Fig. 9h). We were surprised to find that $\mathrm{CNO}$ injection completely blocked the development of CPN ligation-induced neuropathic pain-like hypersensitivity in mice expressing hM3Dq, whereas $\mathrm{CNO}$ injection in control mice transfected with mCherry had no effect on the $\mathrm{CPN}$-induced neuropathic pain-like hypersensitivity for up to 4 weeks, as assayed by the PWT (Fig. 9i). Consistently, we found that prolonged pharmacogenetic activation of GABAergic LPBN neurons reduced the CPN ligation-induced c-Fos expression (Fig. 9j, k). These results demonstrate that prolonged activation of GABAergic LPBN neurons during the first week of CPN ligation prevents the development of neuropathic pain-like hypersensitivity.

\section{Discussion}

Our study, using both gain-of-function and loss-of-function approaches, demonstrates that an LPBN circuit modulates both the sensory and emotional components of neuropathic pain-like hypersensitivity, consistent with the hypothesis that the LPBN not only functions as a relay nucleus for transmitting nociceptive signals from the spinal cord to higher centers, but also actively participates in the development of neuropathic pain, a persistent unpleasant experience involving both sensory and aversive emotional components. In this respect, it is interesting to note 
a
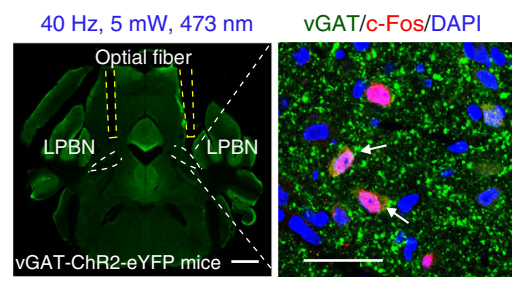

d

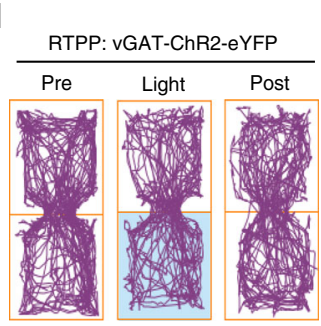

h

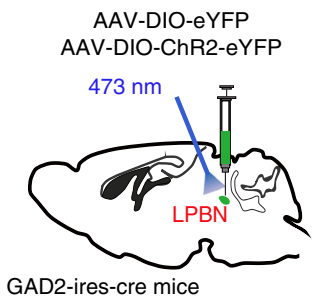

e

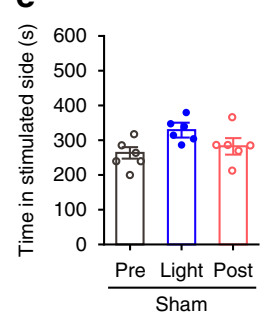

f
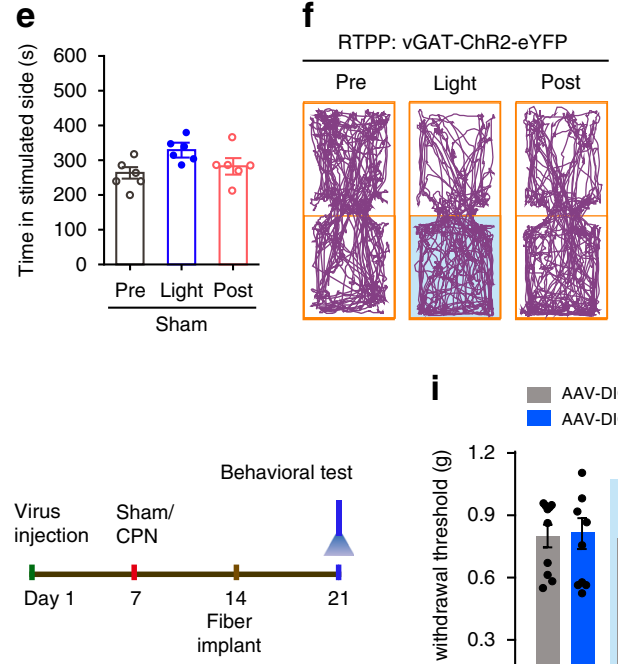

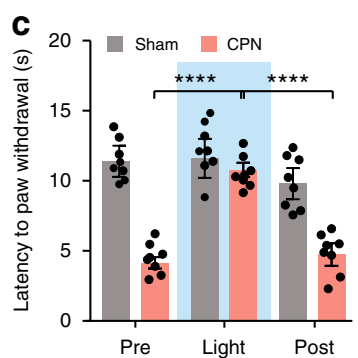

g

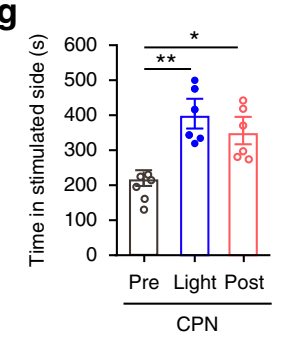

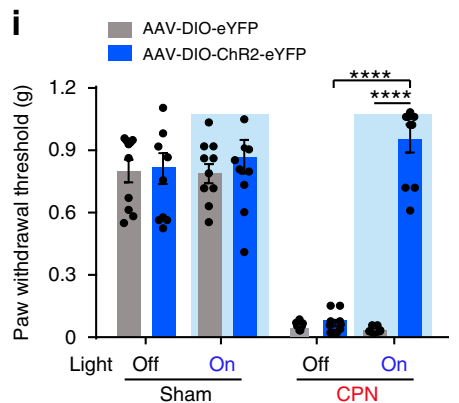

j

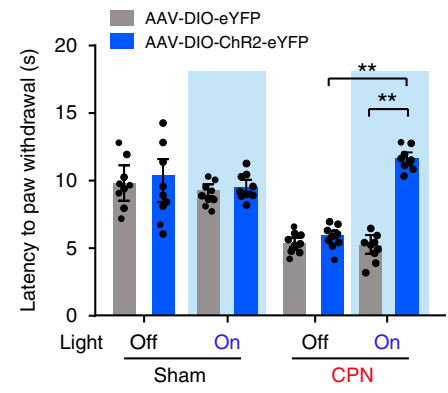

$\mathbf{k}$
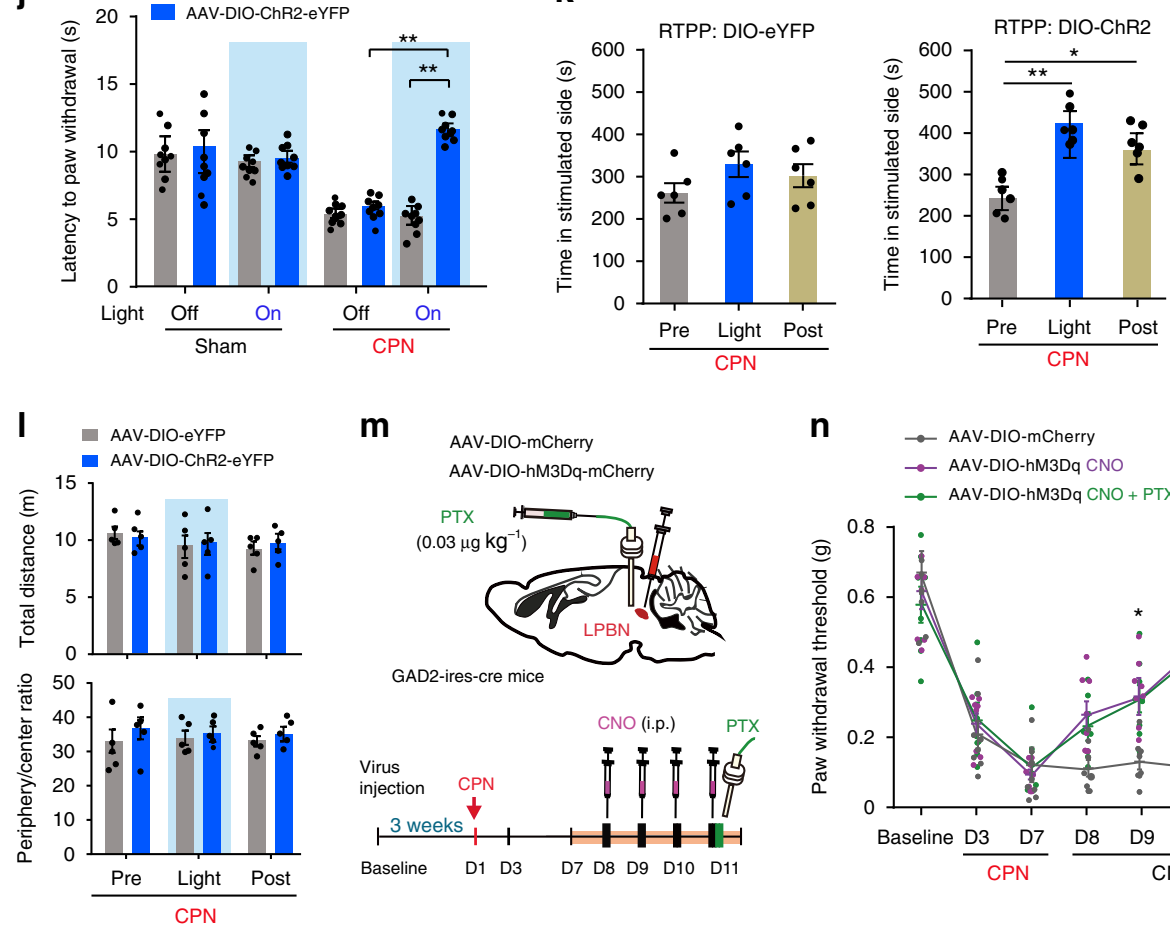

m

AAV-DIO-mCherry

AAV-DIO-hM3Dq-mCherry

n

$\rightarrow$ AAV-DIO-mCherry

$\rightarrow$ AAV-DIO-hM3Dq CNO

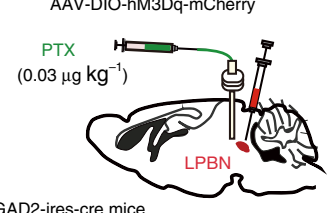

GAD2-ires-cre mice
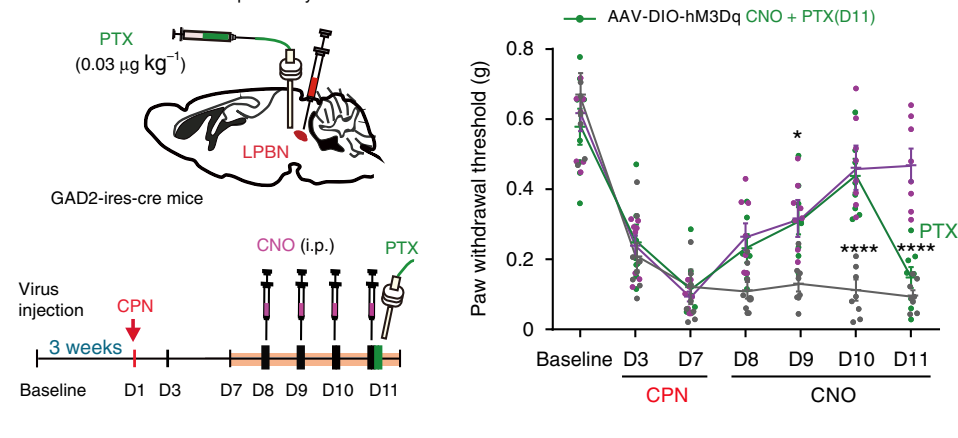

that the PBN has also been shown to regulate various aversive emotional behaviors including threat and avoidance memory ${ }^{31,58}$, anorexia $30,33,34,54$, and escape behavior in response to noxious stimuli $^{59,29}$.

Activation of glutamatergic LPBN neurons is necessary and sufficient to induce neuropathic pain-like behavior. We found here that glutamatergic LPBN neurons were selectively activated in neuropathic pain-like hypersensitivity, as evidenced by 
Fig. 6 Optogenetic stimulation of LPBN GABAergic neurons suppresses neuropathic pain-like hypersensitivity, but not basal nociception. a Image showing optic fibers position in the LPBN (left; scale bar, $1 \mathrm{~mm}$ ) and c-Fos (red) co-labeled with vGAT-positive neurons (green) in the LPBN after optogenetic stimulation (right; arrows, double-labeled neurons; scale bar, $30 \mu \mathrm{m}$ ). b, c Photoactivation of GABAergic LPBN neurons elevate the PWT (b) and thermal paw-withdrawal latency (c) in CPN-ligated, but not in Sham-operated vGAT-ChR2-eYFP mice. d, $\mathbf{f}$ Tracking maps of RTPP from a Sham (d) and a CPN-ligated mouse (f) before, during, and after optogenetic stimulation of GABAergic LPBN neurons. e, $\mathbf{g}$ Quantification of time spent in stimulated side as shown in $\mathbf{d}$ and $\mathbf{f}$. $\mathbf{h}$ Schematic of virus delivery (left) and timeline of the behavioral experiments (right). $\mathbf{i}, \mathbf{j}$ Optogenetic activation of the GABAergic LPBN neurons increased the PWT (i) and thermal paw-withdrawal latency (j) in CPN-ligated mice, but not in Sham-operated mice or in mice transfected with DIO-eYFP. k Quantification of time spent in the non-preferred RTPP chamber before (Pre), during (Light), and after (Post) laser stimulation of CPNligated GAD2-ires-Cre mice transfected with AAV-DIO-eYFP (left) or AAV-DIO-ChR2-eYFP (right). I Quantification of total distance moved (upper) and ratio of time spent in the periphery and center (lower) in the OFT before (Pre), during (Light), and after (Post) light stimulation of GABAergic LPBN neurons in GAD2-ires-Cre mice. $\mathbf{m}$ Experimental design for pharmacogenetic activation of GABAergic LPBN neurons. $\mathbf{n}$ Time-course of the CPN ligationinduced decrease in PWT and the effect of pharmacogenetic activation of GABAergic LPBN neurons which was reversed by administration of PTX ( $0.03 \mu \mathrm{g}$ $\mathrm{kg}^{-1}$ ) via a cannula into the LPBN at day 11. All data are presented as mean \pm s.e.m. and error bars represent s.e.m. ${ }^{\star} P<0.05,{ }^{\star \star} P<0.01$, and ${ }^{\star \star \star \star} P<0.0001$. See also Supplementary Table 1 for further statistical information. Source data are provided as a Source Data file.

increased c-Fos expression (Fig. 1a-d) and the noxious pinch and mechanical stimulation-induced dramatic $\mathrm{Ca}^{2+}$ elevation in glutamatergic (Fig. 1j, k and Supplementary Fig. 2d-g) but not in GABAergic neurons (Fig. $1 \mathrm{~m}, \mathrm{n}$ ) in $\mathrm{CPN}$-ligated mice. Interestingly, the optogenetic activation of glutamatergic LPBN neurons instantly induced neuropathic pain-like symptoms in naïve mice, including mechanical allodynia (Fig. 2d and Fig. 3b) and thermal hyperalgesia (Fig. 2e and Fig. 3c), as well as RTPA behavior (Fig. $2 \mathrm{~g}-\mathrm{j}$ and Fig. $3 \mathrm{~d}$, e), whereas the optogenetic inhibition of glutamatergic LPBN neurons instantly relieved neuropathic painlike hypersensitivity in CPN-ligated mice (Fig. 4b, c and Fig. 5b, c). These results indicate that the activation of glutamatergic LPBN neurons is both necessary and sufficient for the induction and maintenance of neuropathic pain-like behavior. We found that optogenetic activation of LPBNVgluT2 neurons did not further enhance hypersensitivity in CPN-ligated mice (Fig. $2 \mathrm{~d}$, e). It is possibly that CPN treatment already induced hypersensitivity to the maximum extent, which would mask the effects of optogenetic activation of LPBN ${ }^{\text {VluT2 }}$ neurons. These results suggest that the two types of treatments (optogenetic activation of $\mathrm{LPBN}^{\text {VgluT2 }}$ neurons and CPN treatment) share the same mechanisms for inducing hypersensitivity.

We found that prolonged pharmacogenetic activation of glutamatergic LPBN neurons for one week resulted in fullydeveloped neuropathic pain-like hypersensitivity that persisted for several weeks after the termination of pharmacogenetic activation (Fig. 9a, b). This is particularly interesting, since neuropathic pain is characterized as spontaneous discomfort or as painful hypersensitivity to temperature and touch that persists even after recovery from nerve injury ${ }^{1,2,7}$. Structural and functional plasticity induced by nerve trauma leading to the sensitization and increased excitability of peripheral nociceptors or central neurons, especially in the spinal dorsal horn ${ }^{1-3,5,17,60,61}$, have been implicated in the post-injury hypersensitivity. Our findings suggest that the plasticity and sensitization of glutamatergic LPBN neurons induced by prolonged nociceptive signaling following nerve injury is also crucial for the development of persistent neuropathic pain.

The results that optogenetic inhibition of glutamatergic LPBN neurons not only blocked neuropathic pain-like hypersensitivity but also elevated the basal nociception threshold in naïve mice (Fig. 4b, c, and Fig. 5b, c) are consistent with the hypothesis that glutamatergic LPBN neurons are crucial for both relaying physiological pain and mediating neuropathic pain.

GABAergic LPBN neurons selectively gate neuropathic painlike behavior but not basal nociception. It is interesting to note that GABAergic neurons only constitute $\sim 10.1 \%$ of LPBN neurons, much less than that reported in the spinal dorsal horn $(\sim 70 \%)^{62}$ and in the central nucleus of the amygdala $(\sim 50.78 \%)$, but comparable to that reported in the thalamus $(\sim 10.43 \%)$ and somatosensory cortex $(\sim 8.66 \%)$ (Allen Brain Atlas, http://www. brain-map.org) in the pain transmission pathway, suggesting that glutamatergic LPBN neurons that also receive GABAergic innervation from other regions ${ }^{28,54,53}$ are under the relatively weak control of local GABAergic neurons. Consistent with this proposal, we found that the optogenetic activation of GABAergic LPBN neurons did not affect the paw-withdraw response in naïve mice (Fig. 6b, c). However, we found that glutamatergic LPBN neurons receive direct monosynaptic input from local GABAergic neurons, as demonstrated by monosynaptic virus tracing and direct electrophysiological recording (Fig. 8a-k). Interestingly, optogenetic activation of GABAergic LPBN neurons efficiently blocked the mechanical allodynia, thermal hyperalgesia, and affective pain-like behavior in CPN-ligated mice (Fig. 6b-l). Furthermore, the analgesia induced by pharmacogenetic activation of GABAergic LPBN neurons in CPN-ligated mice was reversed by injection of the $\mathrm{GABA}_{\mathrm{A}}$ receptor chloride channel blocker PTX into the LPBN, suggesting that GABAergic LPBN neurons gate neuropathic pain transmission via local inhibition of glutamatergic neurons (Fig. $6 \mathrm{~m}, \mathrm{n}$ ). However, our study does not exclude the possibility that GABAergic input from other brain regions to the LPBN may also play an important role in neuropathic pain-like hypersensitivity. In this respect, it should be noted that a recent study has shown that suppressed GABAergic input from the central nucleus of the amygdala to the LPBN may contribute to chronic pain ${ }^{28}$.

The result that optogenetic activation of GABAergic LPBN neurons did not affect the paw-withdrawal response in naïve mice is in sharp contrast to the effect of direct optogenetic inhibition of glutamatergic LPBN neurons, which not only blocked neuropathic pain-like hypersensitivity but also elevated the basal nociception threshold in naïve mice (Fig. $4 \mathrm{~b}, \mathrm{c}$ and $5 \mathrm{~b}, \mathrm{c}$ ). One possibility for the discrepancy between the result of optogenetic inhibition of glutamatergic LPBN neurons and that of optogenetic activation of GABAergic LPBN neurons may be that the former, relaying physiological pain, may receive relatively weak inhibition from local GABAergic neurons, while the development of neuropathic pain may recruit an additional population of glutamatergic neurons that are normally under tighter control by local GABAergic neurons. In this way, the basic physiological pain signal important for survival can be faithfully relayed without spreading to and sensitizing the glutamatergic LPBN neurons involved in pathological pain. Consistent with this hypothesis, we found that optogenetic inactivation of GABAergic LPBN neurons was sufficient to induce hyperalgesia and RTPA in control mice 
a

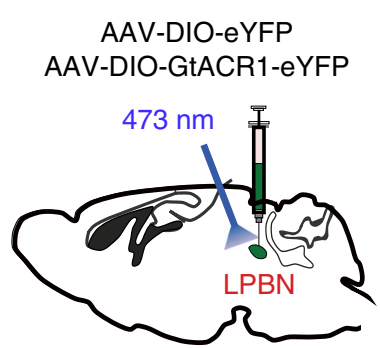

GAD2-ires-cre mice b AAV-DIO-eYFP

AAV-DIO-GtACR1-eYFP

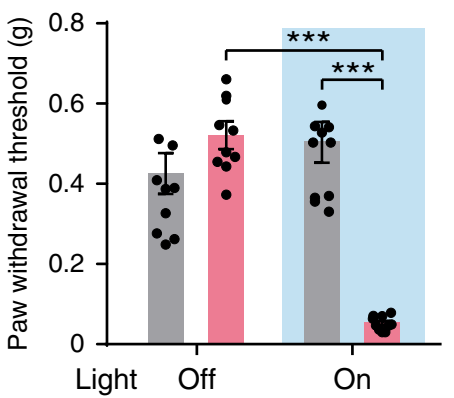

C AAV-DIO-eYFP

AAV-DIO-GtACR1-eYFP

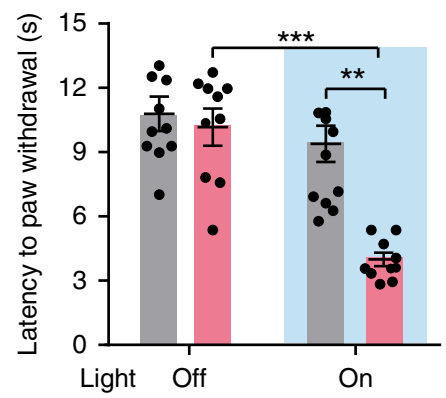

d

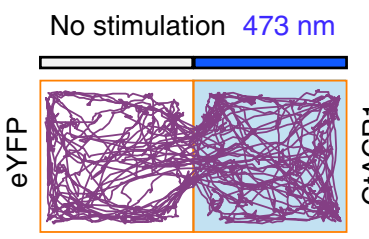

RTPA

No stimulation $473 \mathrm{~nm}$
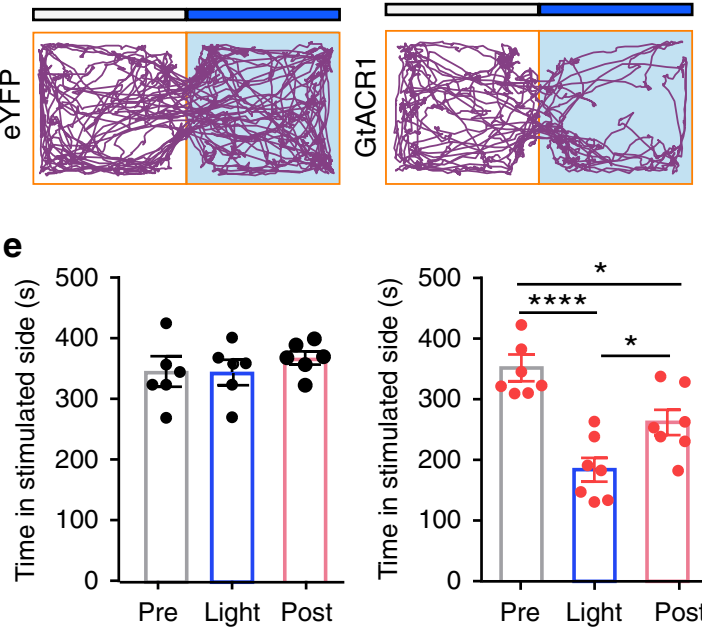

f

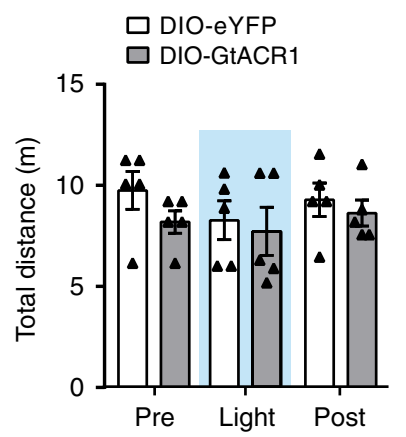

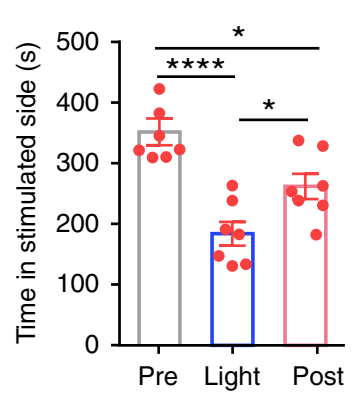

h

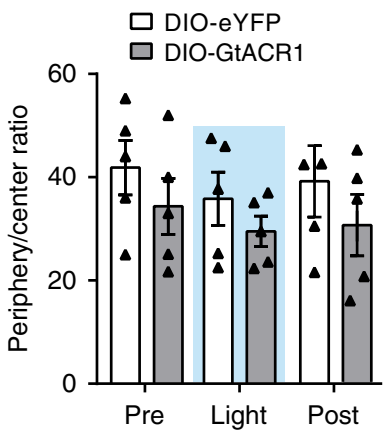

g
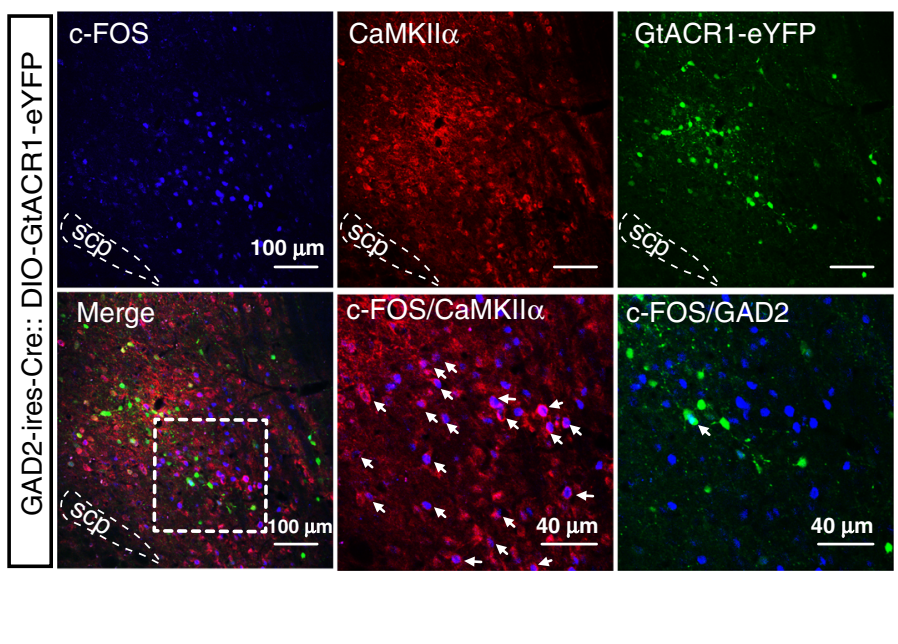

i

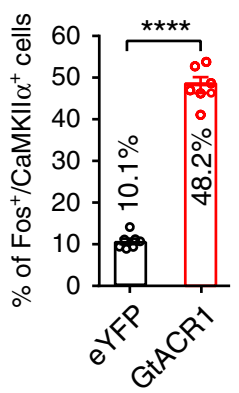

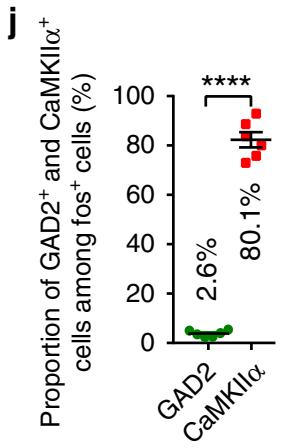

Fig. 7 Optogenetic inhibition of GABAergic LPBN neurons induced neuropathic pain-like symptoms. a Schematic of the stereotaxic delivery of AAVDIO-GtACR1-eYFP and AAV-DIO-eYFP into the LPBN of GAD2-ires-Cre mice and photoinhibition of GABAergic LPBN neurons. b Photoinhibition (473 nm, $40 \mathrm{~Hz}, 5 \mathrm{~mW}$ ) of GABAergic LPBN neurons decreases the PWT in GAD2-ires-Cre mice transfected with AAV-DIO-GtACR1-eYFP. c Photoinhibition of GABAergic LPBN neurons decreases thermal withdraw latency in GAD2-ires-Cre mice transfected with AAV-DIO-GtACR1-eYFP. d Representative tracks in RTPA during 10-min illumination of the LPBN in GAD2-ires-Cre mice transfected with AAV-DIO-eYFP (left) or AAV-DIO-GtACR1-eYFP (right). e Quantification of time spent in the preferred chamber in the RTPA test before (Pre), during (Light), and after (Post) 10-min illumination of the LPBN in GAD2-ires-Cre mice transfected with AAV-DIO-eYFP (left) or AAV-DIO-GtACR1-eYFP (right). $\mathbf{f}$ Quantification of total distance moved (left) and ratio of time spent in the periphery and center (right) in the OFT before (Pre), during (Light), and after (Post) 10-min illumination of GABAergic LPBN neurons in GAD2-ires-Cre mice transfected with AAV-DIO-eYFP or AAV-DIO-GtACR1-eYFP. g Representative images of c-Fos expression (blue) $1 \mathrm{~h}$ after optogenetic inhibition ( $473 \mathrm{~nm}, 5 \mathrm{~mW}, 10 \mathrm{~min}$ ) of GABAergic LPBN neurons in GAD2-ires-Cre mice transfected with AAV-DIO-GtACR1-eYFP. Lower middle and lower right panels show magnified views of the boxed area in the lower left panel. Note that c-Fos-positive cells co-localize with CaMKIl $\alpha$ (red), but not with GtACR1-eYFP (GAD2, green). Arrows indicate CaMKIl $\alpha^{+}$neurons co-labeled with c-Fos. Scale bars, $100 \mu \mathrm{m}$ and $40 \mu \mathrm{m}$ (lower middle and lower right panels). h, i Quantification of cells expressing c-Fos induced by illumination of GABAergic LPBN neurons in GAD2-ires-Cre mice transfected with AAV-DIO-GtACR1-eYFP or AAV-DIO-eYFP as in $\mathbf{g}$. $\mathbf{j}$ Proportion of c-Fos-positive cells that co-express GAD2-eYFP or CaMKIll ${ }^{+}$cells $1 \mathrm{~h}$ after optogenetic inhibition of GABAergic LPBN neurons as shown in $\mathbf{g}$. All data are presented as mean \pm s.e.m. and error bars represent s.e.m. ${ }^{\star} P<0.05,{ }^{\star \star} P<$ $0.01{ }^{\star \star \star} P<0.001$, and ${ }^{\star \star \star \star} P<0.0001$. See also Supplementary Table 1 for further statistical information. Source data are provided as a Source Data file. 
a

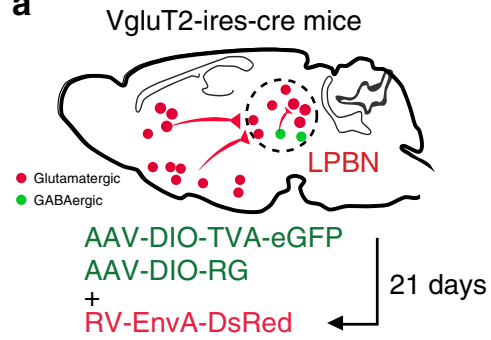

b

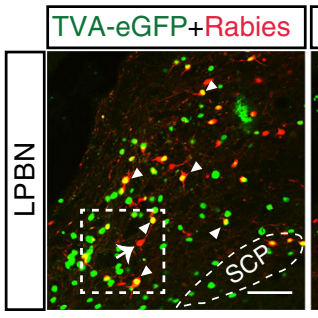

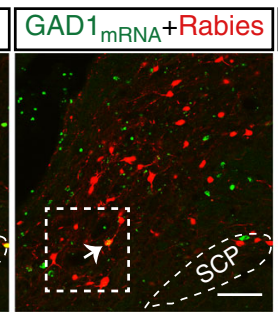

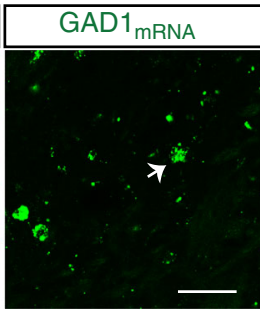

$\mathbf{f}$
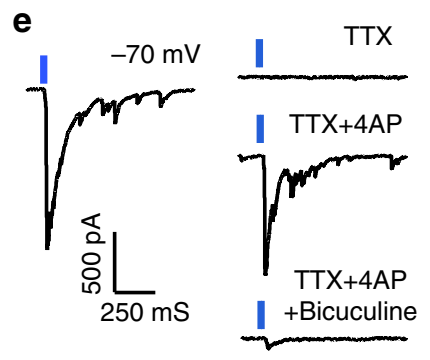

d eYFP/CaMKII $\alpha / D A P I$

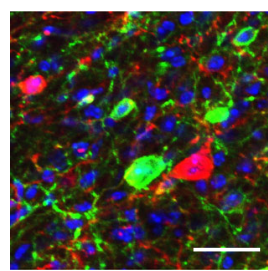

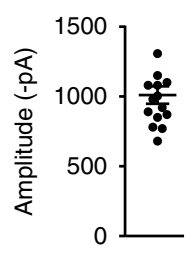

g

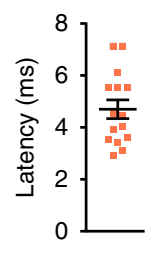

h

$473 \mathrm{~nm}, 20 \mathrm{~Hz}, 5 \mathrm{~ms}$
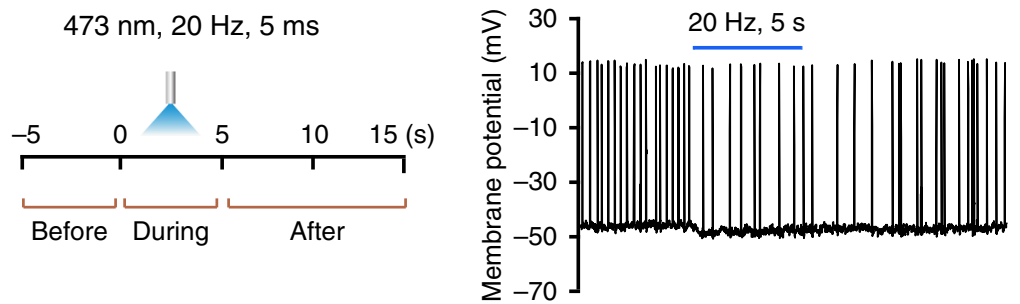

i
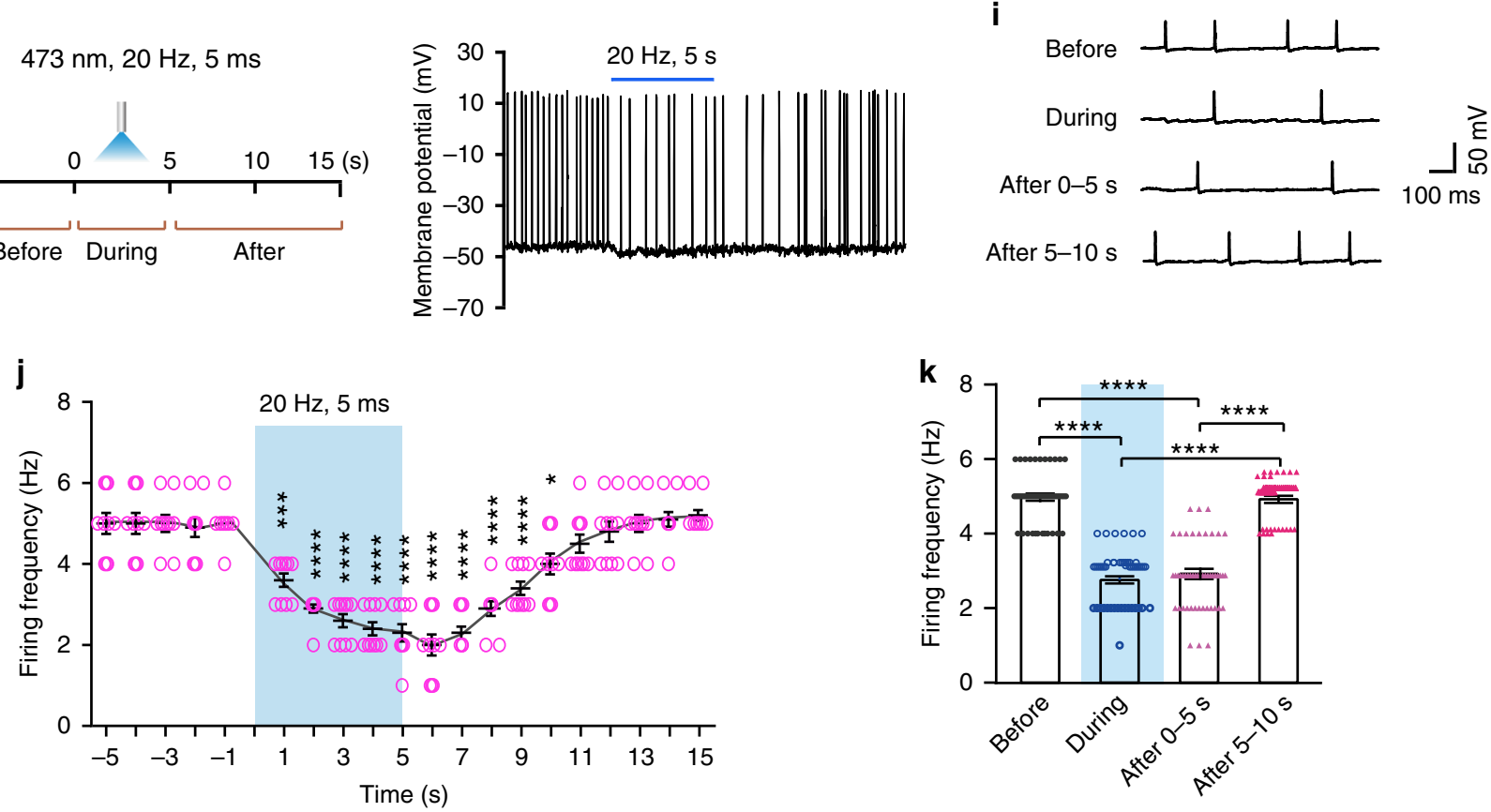

Fig. 8 GABAergic LPBN neurons monosynaptically innervate local glutamatergic neurons. a Schematic of monosynaptic retrograde rabies virus tracing from glutamatergic LPBN neurons. b Representative images showing monosynaptic rabies spread from glutamatergic LPBN neurons in VgluT2-ires-Cre mice. Arrowheads, starter neurons infected by both TVA-eGFP (green) and rabies virus (red). The arrow indicates a GABAergic neuron (labeled with GAD1 mRNA, green) that was trans-synaptically infected by rabies virus. The third and fourth panels from the left show magnified views of the boxed areas in the second panel. Scale bars, $100 \mu \mathrm{m}$ and $30 \mu \mathrm{m}$ (magnified views). c Schematic of patch clamp recording from glutamatergic LPBN neurons transfected with AAV-CaMKIl $\alpha$-mCherry in response to light stimulation of GABAergic LPBN neurons transfected with AAV-DIO-ChR2-eYFP. d Example image showing the expression of ChR2 in GABAergic neurons (green) and mCherry in CaMKIl $\alpha$ neurons (red) for targeted whole-cell patch-clamp recordings as in c. Scale bar, $30 \mu \mathrm{m}$. e Example of light-evoked IPSCs recorded from mCherry-positive glutamatergic neurons at a holding potential of $-70 \mathrm{mV}$ (left panel). The IPSCs were blocked by $1 \mu \mathrm{M}$ TTX, and they were rescued by $100 \mu \mathrm{M} 4$-AP and blocked again by $10 \mu \mathrm{M}$ bicuculline (right panel). $n=12$ cells in 6 slices from 4 mice. $\mathbf{f}, \mathbf{g}$ Averaged amplitude and latency of recorded IPSCs as in $\mathbf{o} . n=15$ traces recorded in 6 slices from 4 mice. $\mathbf{h}$ Workflow (left) and representative current clamp recording trace (right) of spontaneous spikes in an mCherry-positive LPBNCaMKIlo neuron at a holding potential of $-45 \mathrm{mV}$. i Extended traces from (h) with a slow time scale showing light-induced changes in spike frequency of an LPBNCaMKIl $\alpha$ neuron sampled before, during, and after light stimulation of GABAergic LPBN neurons. $\mathbf{j}$ Time course of summarized changes in spike frequency of LPBN CaMKIll $\boldsymbol{\alpha}$ neurons induced by light stimulation of GABAergic LPBN neurons. $\mathbf{k}$ Averaged firing frequency of LPBN CaMKIll $\alpha$ neurons during each period as indicated. All data are presented as mean \pm s.e.m. and error bars represent s.e.m. ${ }^{\star} P<0.05,{ }^{\star \star} P<0.01$, and ${ }^{\star \star \star \star}{ }^{\star} P<0.0001$. See also Supplementary Table 1 for further statistical information. Source data are provided as a Source Data file. 
a

VgluT2-ires-cre mice:
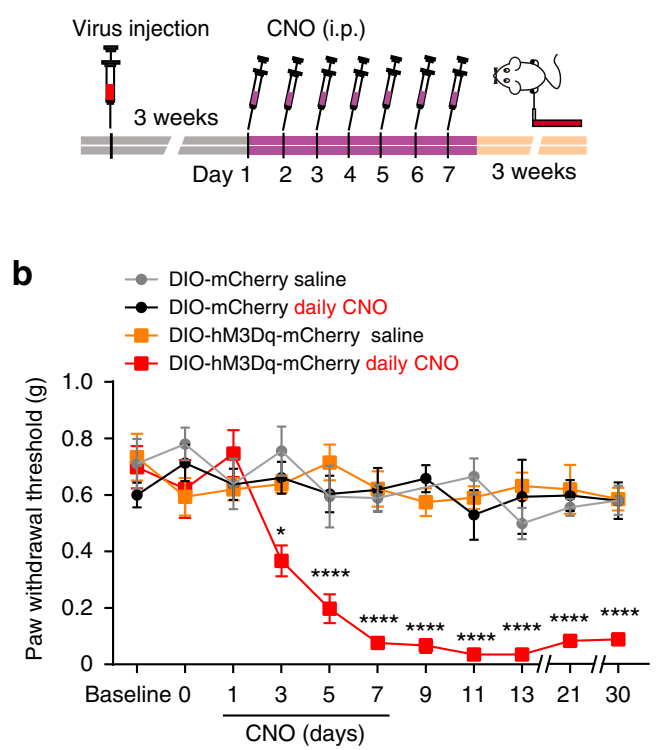

f GAD2-ires-cre mice:

Virus
injection $\quad$ CNO (i.p.)

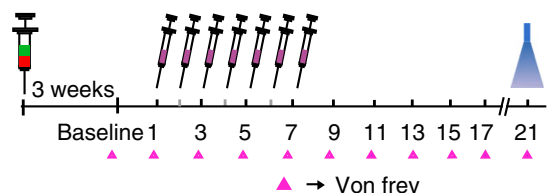

g
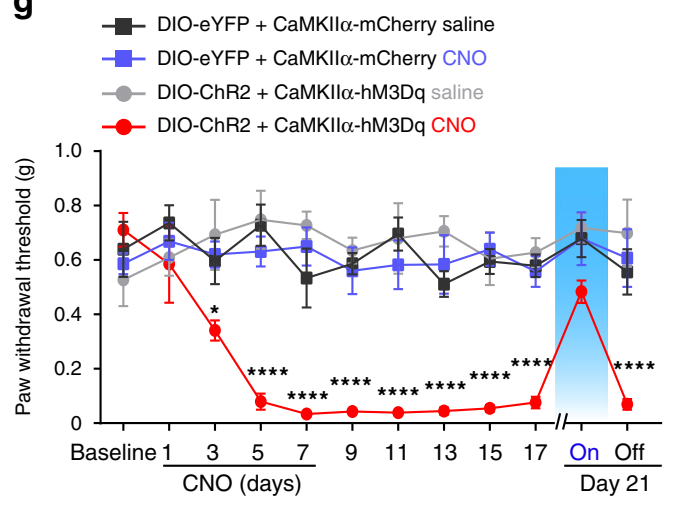

c

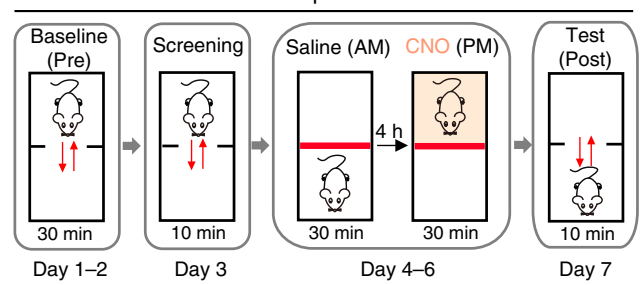

d
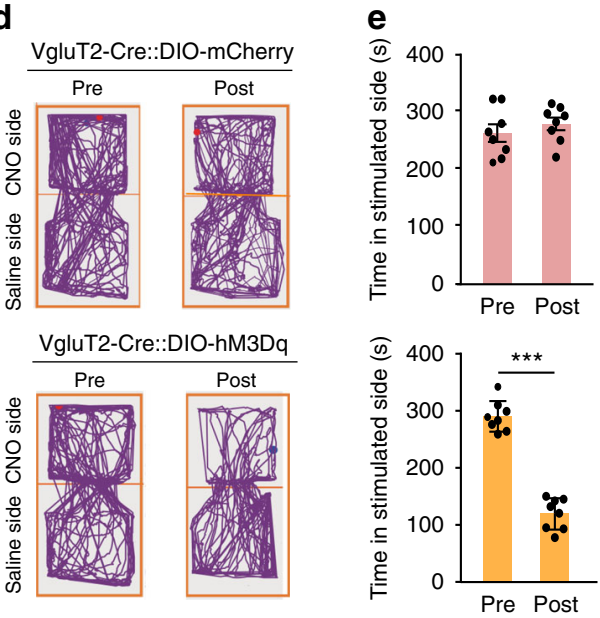

h

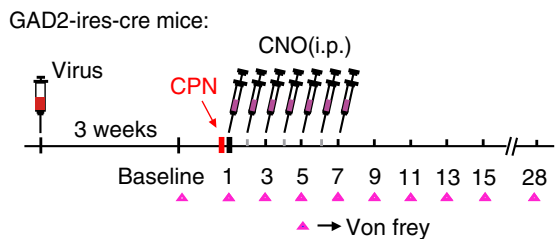

i

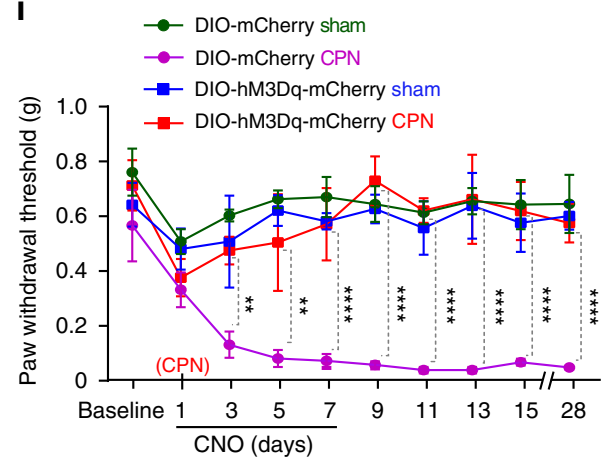

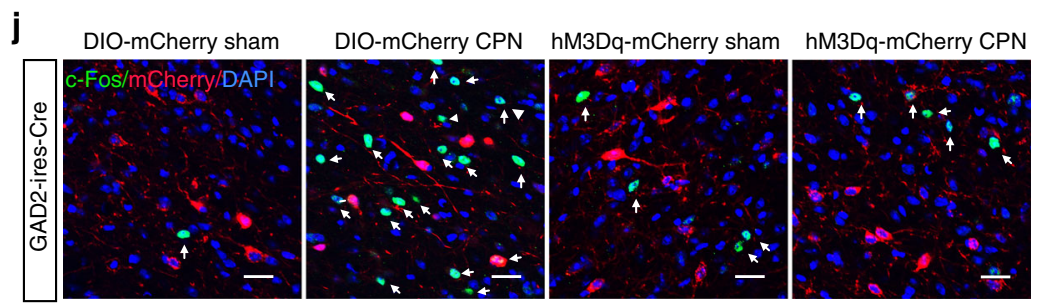

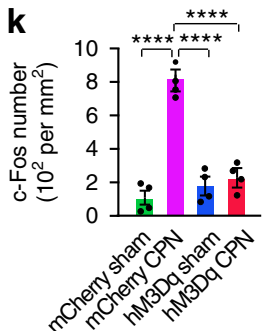

mimicking neuropathic pain (Fig. 7a-e). It is interesting to note that the activation of hunger-sensitive and $\mathrm{PBN}$-projecting arcuate nucleus neurons that co-express agouti-related protein, neuropeptide $Y$, and GABA attenuates inflammatory pain-like hypersensitivity without altering acute nociceptive responses ${ }^{33}$.
Development of neuropathic pain-like hypersensitivity is due to an imbalance of excitatory-inhibitory neuronal activity in the LPBN circuit. We found that most of the cells expressing cFos induced by CPN ligation $(\sim 74.8 \%)$ were glutamatergic (constituting $\sim 41.8 \%$ of all glutamatergic neurons), while only 


\section{Fig. 9 Persistent activation of glutamatergic and GABAergic LPBN neurons, respectively, develops and prevents neuropathic pain-like}

hypersensitivity. a Experimental design and timeline of the behavioral experiment. b Time-course of PWT changes induced by daily injection of CNO (i.p., $1.5 \mathrm{mg} \mathrm{kg}^{-1}$ ) for one week in VgluT2-ires-Cre mice. c Schematic of the experiment design for conditioned place aversion (CPA). $\mathbf{d}$ Examples of tracking maps in the CPA before (Pre) and after (Post) injection of CNO (i.p., $1.5 \mathrm{mg} \mathrm{kg}^{-1}$ ) into the LPBN of VgluT2-ires-Cre mice transfected with DIO-mCherry (upper) or DIO-hM3Dq-mCherry (lower). e Quantification of time spent in the preferred chamber as in $\mathbf{d}$. $\mathbf{f}$ Experimental design and timeline of the behavioral experiment in GAD2-ires-Cre mice simultaneously transfected with two types of virus in the LPBN, one carrying DIO-eYFP (control) or DIOChR2-eYFP and the other carrying CaMKIl $\alpha$-mCherry (control) or CaMKIl $\alpha$-hM3Dq-mCherry. $\mathbf{g}$ Time-course of PWT changes induced by daily injection of CNO (i.p., $1.5 \mathrm{mg} \mathrm{kg}^{-1}$ ) for one week in GAD2-ires-Cre mice. A persistent decrease in PWT was induced only in mice injected with CNO and transfected with CaMKIl $\alpha-h M 3 D q-m C h e r r y$, but not in the other groups. Note that illumination ( $473 \mathrm{~nm}, 40 \mathrm{~Hz}, 5 \mathrm{~mW}$ ) of GABAergic LPBN neurons on day 21 reversibly elevated the PWT in mice transfected with DIO-ChR2-eYFP. $\mathbf{h}$ Experimental design and timeline of the behavioral experiment. $\mathbf{i}$ Daily injection of CNO for one week initiated on the same day as CPN ligation prevented the development of neuropathic pain-like hypersensitivity in mice transfected with DIO-hM3Dq-mCherry (red), but not in mice transfected with DIO-mCherry. j Representative images showing c-Fos expression (green, arrows) and GABAergic neurons transfected with mCherry or hM3Dq-mCherry (red) in the LPBN from Sham-operated and CPN-ligated mice. Scale bar, $30 \mu \mathrm{m}$. Blue, DAPI stain). $\mathbf{k}$ Quantification of c-Fos-positive cells in the LPBN from different groups as in $\mathbf{j}$. All data are presented as mean \pm s.e.m. and error bars represent s.e.m. ${ }^{\star} P<0.05,{ }^{\star \star} P<0.01$, and ${ }^{\star \star \star \star} P<0.0001$. See also Supplementary Table 1 for further statistical information. Source data are provided as a Source Data file.

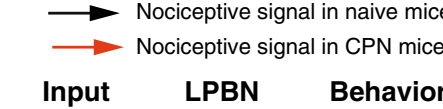

a Activation of glutamatergic neuron

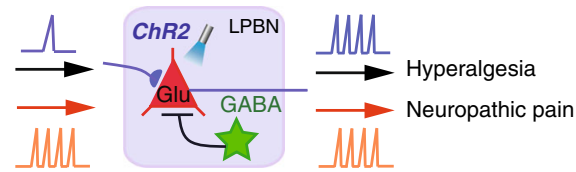

b

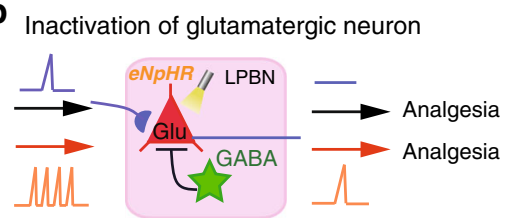

C Repetitive activation of glutamatergic neuron

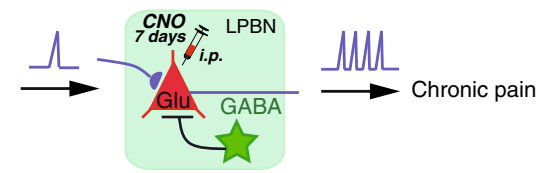

Input LPBN Behavior

d Activation of GABergic neuron

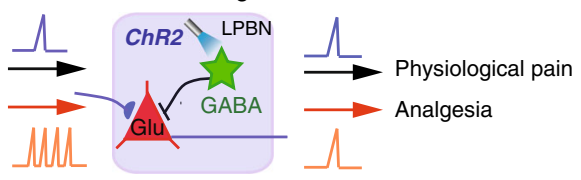

e

Inactivation of GABergic neuron

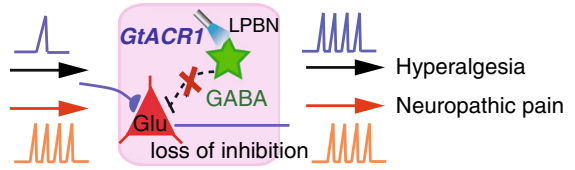

f Repetitive activation of GABergic neuron

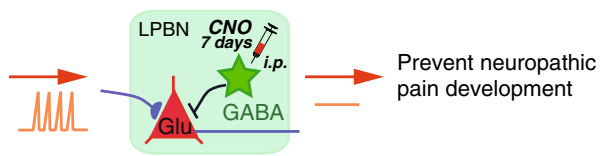

Fig. 10 Schematic summary of the main findings illustrating how activity in the LPBN circuit governs physiological and pathological pain-like

hypersensitivity. a Light-activation of glutamatergic neurons induces hyperalgesia in naive mice to an extent similar to that in CPN-ligated mice. $\mathbf{b}$ Lightinactivation of glutamatergic neurons induces analgesia in both naive and CPN-ligated mice. c Daily pharmacogenetic activation of glutamatergic neurons for one week induces chronic nociception, mimicking neuropathic pain. $\mathbf{d}$ Light-activation of GABAergic neurons inhibits neuropathic pain-like hypersensitivity, but not basal nociception. e Light-inactivation of GABAergic neurons induces hyperalgesia in naive mice to an extent similar to that in CPN-ligated mice. $\mathbf{f}$ Daily pharmacogenetic activation of GABAergic neurons for one week prevents the development of neuropathic pain-like hypersensitivity in CPN-ligated mice.

$2.1 \%$ of the c-Fos-positive cells were GABAergic (constituting $\sim 8.5 \%$ of all GABAergic neurons). These results, together with the finding that after CPN ligation, pinch stimulation induced dramatic $\mathrm{Ca}^{2+}$ elevation in glutamatergic, but not GABAergic neurons (Fig. $1 \mathrm{i}-\mathrm{n}$ ), suggest that glutamatergic neurons are selectively activated and sensitized by CPN ligation. Thus, the robust nociceptive signaling from the spinal cord due to prolonged noxious stimulation such as CPN ligation may upset the excitatoryinhibitory balance for a sufficient time within the LPBN circuit and lead to the development of neuropathic pain-like hypersensitivity (Fig. 10).

The results that optogenetic activation of GABAergic LPBN neurons instantly relieved neuropathic pain-like hypersensitivity (Fig. $6 \mathrm{~b}, \mathrm{c}, \mathrm{i}, \mathrm{j}$ ) and that prolonged pharmacogenetic activation of these neurons at an early stage of CPN ligation completely blocked the development of neuropathic pain-like hypersensitivity (Fig. 9i) indicate that they not only control the transmission but also govern the development of neuropathic pain. Prolonged activation of GABAergic LPBN neurons may restore the balance of excitation and inhibition in the LPBN circuit and prevent the sensitization of glutamatergic neurons induced by $\mathrm{CPN}$ ligation (Fig. 10). Indeed, we found that repetitive pharmacogenetic activation of GABAergic LPBN neurons prevented the increased c-Fos expression in the LPBN induced by CPN ligation (Fig. 9j, k). It has been reported that the development (early phase) and maintenance (late phase) of neuropathic pain may have distinct mechanisms ${ }^{63-65}$. It will be interesting to determine whether GABAergic LPBN neurons also play a critical role in the 
late phase of neuropathic pain-like hypersensitivity (after 3 weeks).

The densely-distributed GABAergic and glycinergic interneurons in the dorsal horn have been regarded as a key component of the gate control theory of pain ${ }^{12,60,66,67}$. Disinhibition due to the selective loss of GABAergic interneurons or collapse of the $\mathrm{Cl}^{-}$ gradient in the dorsal horn neurons mediated by glial cell-derived BDNF and cytokines has been proposed as a mechanism of opening the gate for pain ${ }^{12,68-70}$. It is unclear if glial cells and cytokines also play a role in the LPBN in neuropathic pain. We found that CPN treatment did not change the density of GABAergic LPBN neurons, although the release probability of these neurons decreased (Supplementary Fig. 6a-e). Our study demonstrates that the balance of excitation and inhibition in the LPBN circuit may provide another important gate control of neuropathic pain. Unlike in the dorsal horn, however, GABAergic neurons constitute only a very small population in the LPBN. Due do the relatively weak GABAergic control of the LPBN circuit, selective activation of glutamatergic over GABAergic neurons in the LPBN together with a decreased release probability of GABAergic LPBN neurons by nerve injury is thus sufficient to break this delicate excitatory-inhibitory balance, leading to loss of gate control and the development of neuropathic pain (Fig. 10).

Sex dimorphism has been reported in some pain mechanisms ${ }^{71-73}$. In particular, a recent study reported that neuropathic pain is accompanied by sex-specific changes in the excitability and synaptic function of different subtypes of GABAergic neurons in prelimbic cortex ${ }^{72}$. We only used male mice here and it would be interesting to determine in future whether similar sex-specific changes occur in LPBN neurons in neuropathic painlike hypersensitivity.

The intricate relationship between pain and affective and emotional responses has been well studied, and the amygdala, which receives nociceptive signals directly from the $\mathrm{PBN}^{18,74,75}$, is regarded as a key node in processing the affective and emotional components of pain ${ }^{4,14,35,76}$. The sustained nociceptive signaling caused by nerve injury may result in structural and functional plasticity in the spinoparabrachial pathway. The sensitized glutamatergic LPBN neurons may then convey sensory signals to the amygdala with heightened sensitivity and associate the neuropathic pain with aversive emotional responses (Fig. 10). It is also possible that LPBN modulates neuropathic pain through activation of descending modulation pathways, such as basolateral amygdala-prefrontal cortex-periaqueductal gray (PAG)spinal cord pathway reported recently ${ }^{77}$.

Neuropathic pain remains a major public health burden and effective therapy is still lacking ${ }^{78}$. Since neuropathic pain also happens in in supraspinal CNS injury or brain disorders ${ }^{1,2}$, higher brain regions also play crucial roles in neuropathic pain development. Our findings suggest that the LPBN is a potential key target for neuropathic pain therapy.

\section{Methods}

Animals. In all experiments, vGAT-ChR2 (H134R)-eYFP (vGAT) (JAX014548), VgluT2-ires-Cre (Slc17a6 ${ }^{\mathrm{tm} 2(\mathrm{cre}) \text { Lowl } / J)}$ (JAX016963), GAD2-ires-Cre (B6N.Cg-

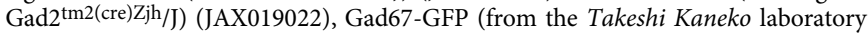
of Kyoto University), and wild-type (C57BL/6J, from the Shanghai SLAC Laboratory Animal CO. LTD) male mice 8-10 weeks of age (20-35 g) were used. All experiments were conducted in accordance with the guidelines for the care and use of laboratory animals of Zhejiang University (ZJU) and were approved by the Animal Advisory Committee at ZJU. The mice were housed 4 or 5 per cage at constant room temperature $\left(25 \pm 1^{\circ} \mathrm{C}\right)$ and relative humidity $(60 \pm 5 \%)$ under a 12 h light/dark schedule (light 07:00-19:00) with food and water ad libitum. All behavioral tests were carried out during the light phase. In the behavioral tests, the mice were allowed to adapt to laboratory conditions for $\sim 1$ week and to habituate to the testing situation for at least $30 \mathrm{~min}$ before experiments. Littermate mice were split into random groups before virus injections. Biohazard wastes were managed
} following the Medical Waste Management Regulations of China.
CPN ligation surgeries. The common peroneal nerve (CPN) ligation model of neuropathic pain has been used ${ }^{39}$. Briefly, mice were anesthetized with sodium pentobarbital ( $1 \% \mathrm{wt} / \mathrm{vol})$. The left common peroneal nerve between the anterior and posterior groups of muscles was ligated slowly with 5-0 chromic gut suture (Ethicon) until twitching of the digits was observed. The skin was sutured with 5-0 silk suture and cleaned with Povidone-iodine. Sham surgery was conducted in the same manner, but the nerve was not ligated. All mice were kept in a normal living cage after surgery.

Measurement of Hypersensitivity Threshold in Mice. Mice should have 3 days of acclimation in all the behavior tests. Measurements of mechanical and thermal withdrawal threshold/latency were performed ${ }^{43}$. The Von Frey test was used to assess the onset and maintenance of mechanical allodynia over time ${ }^{46}$. Dynamic tactile allodynia testing was assessed by lightly stroking the lateral external side of the surface of the injured hindpaw, in the direction from heel to toe with a von Frey monofilament without bending the filament. After acclimatizing the mice for about $60 \mathrm{~min}$ in the observation chambers on top of a grid mesh, the ipsilateral paws were tested 10 times, with intervals of 10-15 s, and the percentage of withdrawal for ipsilateral paw (nerve-injured side) was considered as the hypersensitivity response. Mice were tested before blue or yellow light stimulation (light Off) and after $45 \mathrm{~min}$ they were stimulated with blue or yellow light on (light On) for $3 \mathrm{~min}$ and the ipsilateral hindpaws were tested. The stimulus producing a $50 \%$ likelihood of a withdrawal response was determined and taken as the paw withdrawal threshold $(\mathrm{PWT})^{46,79}$.

The Hargreaves test is specifically designed to assess thermal pain sensation in rodents ${ }^{80}$. Each mouse was placed in a plastic cylinder on a glass floor. A radiant heat beam was focused onto the injured hindpaw (plantar test apparatus from IITC Life Science Inc.). The latency to hindpaw withdrawal of the ipsilateral paw (nerveinjured side) was recorded with at least 3 trials per animal repeated 5 min apart. Beam intensity was adjusted so that control mice displayed a latency of 8-12 s. A cut-off latency of $30 \mathrm{~s}$ was set to avoid tissue damage. Measurement of hypersensitivity threshold was performed during the light cycle by the same researcher who was blinded to the surgery, genotypes, and treatments (CNO versus saline).

Stereotaxic injection. To manipulate the activity of LPBN neurons, adenoassociated viruses (AAVs) were injected bilaterally into the LPBN (AP, $5.02 \mathrm{~mm}$; $\mathrm{ML}, \pm 1.25 \mathrm{~mm}$; DV, $3.27 \mathrm{~mm}$ ) of $8-10$ week-old mice and behavioral tests were performed 2-3 weeks later. All injections were performed with a stereotaxic apparatus on mice under anesthesia with sodium pentobarbital ( $1 \% \mathrm{wt} / \mathrm{vol})$, and body temperature maintained with a heating pad. Ophthalmic ointment was applied to maintain eye lubrication. Viruses were injected at $15 \mathrm{nl} / \mathrm{min}$ using an air pressure system connected to a glass pipette (tip diameter 10-30 $\mu \mathrm{m}$ ). After injection, the pipette was left in place for 15-20 min to allow diffusion. The mice were allowed to recover from anesthesia on a heating blanket before returning to the home cage. Antibiotic (Ceftriaxone sodium, $0.1 \mathrm{~g} / \mathrm{kg}$ ) was injected intraperitoneally daily for three days after surgery to prevent infection.

Viral injection and optical fibers/cannula implantation. Mice were anesthetized with pentobarbital sodium $(0.1 \mathrm{~g} / \mathrm{kg})$ and mounted in a stereotaxic apparatus. The skull was exposed by midline scalp incision, and a craniotomy was performed unilaterally or bilaterally for introduction of a microinjection glass pipette into the LPBN (AP, $5.02 \mathrm{~mm}$; ML, $\pm 1.25 \mathrm{~mm}$; DV, $3.27 \mathrm{~mm}$ ) in a volume of $80-100 \mathrm{nl}$ for each side at $0.05 \mu \mathrm{min}^{-1}$. The syringe was not removed until 15-20 min after the end of infusion to allow diffusion of the virus. A craniotomy window $(\sim 1.5 \mathrm{~mm}$ diameter) over the target area was made using a hand-held drill ${ }^{41,81}$.

For fiber photometry, $100 \mathrm{nl}$ of AAV2/9-hSyn-DIO-GCaMP7s-WPRE (1.34 $\times$ $10^{13}$ genomic copies per ml) was unilaterally injected into the LPBN. After injection of AAV2/9-DIO-GCaMP7s, an optical fiber (outer diameter (OD) of 200 $\mu \mathrm{m}$, numerical aperture (NA) of 0.37 , purchased from Inper) was placed the injection site. For control fiber photometry experiments, $100 \mathrm{nl}$ of AAV2/9-EF1aDIO-eYFP $\left(1.0 \times 10^{12}\right.$ genomic copies per $\left.\mathrm{ml}\right)$ was used instead of GCaMP7s.

For local optogenetic stimulation, 80-100 nl of AAV2/9-CaMKIIa-hChR2 (H134R)-mCherry $\left(1.97 \times 10^{13}\right.$ genomic copies per ml), AAV2/8-EF1a-DIO-ChR2 (H134R)-mCherry $\left(1.25 \times 10^{12}\right.$ genomic copies per ml), AAV2/9-EF1 $\alpha$-DIO-eYFP $\left(1.0 \times 10^{12}\right.$ genomic copies per ml), AAV2/8-EF1 $\alpha$-DIO-mCherry $\left(1.1 \times 10^{12}\right.$ genomic copies per ml), AAV2/8-CaMKII $\alpha$-mCherry $\left(2.13 \times 10^{12}\right.$ genomic copies per ml) was unilaterally injected into the LPBN.

For local photoinhibition stimulation, AAV2/9-CaMKIIa-eNpHR3.0-mCherry $\left(8.3 \times 10^{12}\right.$ genomic copies per ml) or AAV2/8-CaMKIIa-mCherry $\left(2.13 \times 10^{12}\right.$ genomic copies per ml); AAV2/9-EF1 $a$-DIO-eNpHR3.0-eYFP $\left(1.3 \times 10^{13}\right.$ genomic copies per $\mathrm{ml})$ or AAV2/9-EF1a-DIO-eYFP $\left(1.0 \times 10^{12}\right.$ genomic copies per $\left.\mathrm{ml}\right)$; AAV2/9-EF1 $\alpha$-DIO-hGtACR1-P2A-eYFP-WPRE $\left(5 \times 10^{13}\right.$ genomic copies per $\mathrm{ml})$ or AAV2/9-EF1a-DIO-eYFP $\left(1.0 \times 10^{12}\right.$ genomic copies per ml) was bilaterally injected into the LPBN.

For pharmacogenetic stimulation, AAV2/9-hSyn-DIO-hM3Dq-mCherry $\left(2.04 \times 10^{12}\right.$ genomic copies per ml $)$ or AAV2/8-EF1 $\alpha$-DIO-mCherry $\left(1.1 \times 10^{12}\right.$ genomic copies per ml); AAV2/9-CaMKIIa-hM3Dq-mCherry $\left(4 \times 10^{13}\right.$ genomic copies per ml) or AAV2/8-CaMKIIa-mCherry $\left(2.13 \times 10^{12}\right.$ genomic copies per $\left.\mathrm{ml}\right)$ 
was bilaterally injected into the LPBN. These viruses were made by SunBio Biomedical technology Co. or Taitool Bioscience Co. Ltd (Shanghai, China).

For monosynaptic retrograde tracing, $60-80 \mathrm{nl}$ of a mixture (1:1) of AAV2/9CAG-DIO-TVA-eGFP $\left(1.7 \times 10^{13}\right.$ genomic copies per ml $)$ and AAV2/9-CAGDIO-RG $\left(6.8 \times 10^{12}\right.$ genomic copies per $\left.\mathrm{ml}\right)$ was unilaterally injected into the LPBN. We injected the 80-100 nl EnvA-pseudotyped, glycoprotein (RG)-deleted and DsRed-expressing rabies virus (RV-EvnA-DsRed) $\left(5.0 \times 10^{8}\right.$ genomic copies/ $\mathrm{ml}$ ) into the same site 3 weeks later (BrainVTA, China). For the behavioral experiments, animals were allowed to recover for 3-6 weeks, then they were handled and habituated to the behavioral environment every day for one week. For all experiments, mice with incorrect injection sites were excluded from further analysis.

For optogenetic manipulation in awake behaving mice, optical fibers (NA 0.37) were implanted to target the LPBN bilaterally (AP, $5.02 \mathrm{~mm}$; ML, $\pm 1.57 \mathrm{~mm}$; DV, $2.97 \mathrm{~mm}$ at an angle of $5^{\circ}$ ) two weeks after injection of AAV into the LPBN. The fibers (diameter, $200 \mu \mathrm{m}$; length: $6.0 \mathrm{~mm}$ ) were attached to the skull with dental cement and connected to a laser source using an optical fiber sleeve. The power of the blue $(473 \mathrm{~nm}, 5-10 \mathrm{~mW})$ or yellow laser $(589 \mathrm{~nm}, 1 \mathrm{~Hz}, 999 \mathrm{~ms}, 10 \mathrm{~mW})$ (Newdoon Inc., Hangzhou, China) was $0.83-3.33 \mathrm{~mW} \mathrm{~mm}^{-2}$ as measured at the tip of the fiber. GABA neurons were activated by blue light pulses $(10 \mathrm{~ms})$ at 40 $\mathrm{Hz}^{41}$. Glutamatergic neurons were activated by blue light pulses $(5 \mathrm{~ms})$ at $20 \mathrm{~Hz}$, and inhibited using continuous yellow light. Mice with missed injection or cannula locations were excluded. Control mice underwent the same procedures and received the same intensity of laser stimulation.

For pharmacologic experiments, a cannula (RWD Life Science) was implanted above the LPBN (AP, $5.02 \mathrm{~mm}$; ML, $\pm 1.57 \mathrm{~mm}$; DV, $2.97 \mathrm{~mm}$ relative to bregma) for infusion of the $\mathrm{GABA}_{\mathrm{A}}$ receptor blocker PTX $\left(0.03 \mu \mathrm{g} \mathrm{kg}^{-1}\right)$. Two stainless steel screws were implanted in the skull for support. The cannula and screws were held in place with dental cement. A stainless steel obturator was inserted into each guide cannula to prevent blockage. Only mice with the correct locations of optical fibers/ cannulae and viral expression were used for further analysis.

For prolonged activation of glutamatergic and GABAergic LPBN neurons, DREADD activation was achieved in VgluT2-ires-Cre mice and GAD2-ires-Cre mice where AAV-DIO-hM3Dq-mcherry was injected into the LPBN. Clozapine Noxide (CNO, Enzo Life Sciences, Inc.) was dissolved in saline to a concentration of $0.5 \mathrm{mg} / \mathrm{ml}$. In Fig. 7, CNO/saline was injected daily over the first week (a total of 7 injections). Consecutive $\mathrm{CNO}$ (i.p., $1.5 \mathrm{mg} \mathrm{kg}^{-1}$ ) injections were spaced $24 \mathrm{~h}$ apart and mechanical threshold was measured $1 \mathrm{~h}$ before each CNO injection (or $23 \mathrm{~h}$ after previous $\mathrm{CNO}$ injection). No more $\mathrm{CNO}$ injection was given after the first week.

Fiber photometry and endoscopic in vivo calcium imaging. In order to monitor the neuronal activity of glutamatergic LPBN neurons and GABAergic LPBN neurons after AAV-hSyn-DIO-GCaMP7s-WPRE or AAV-EF1 $\alpha$-DIO-eYFP virus were injected into the LPBN of VgluT2-ires-Cre mice and GAD2-ires-Cre mice. After two-three weeks, an optical fiber $(230 \mu \mathrm{m}$ OD, $0.37 \mathrm{NA})$ was implanted into the LPBN, then each mouse was allowed to recover for 3 weeks before recording. Each mouse was handled for 3 days prior to fiber photometry recording. On day 7 , the PWT and $\mathrm{Ca}^{2+}$ transient (or eYFP) signal were recorded simultaneously with F-scope-G-2 after the hindpaw was stimulated with pinch. For noxious mechanical stimulation (pinch), an alligator clip (Amazon, Generic Micro Steel Toothless Alligator Test Clips 5AMP) producing $340 \mathrm{~g}$ force was applied to the ventral skin surface between the footpad and the heel ${ }^{82}$.

A fiber photometry system (Thinker Tech Nanjing Bioscience Inc.) was used for recording 40,83 . At the end of the experiment, all animals were perfused. Only data from animals with correct optical fiber implantation sites and virus expression were included in the analysis. The onset and offset points of stimulation were determined by analyzing the videos frame-by-frame or retrieving analog signals with the MatLab R2016a program. After subtracting the noise of the photometry recording system, we smoothed the data with a moving average filter $(100 \mathrm{~Hz})$. The change in $\mathrm{Ca}^{2+}$ transient value $(\Delta F / F)$ from $2 \mathrm{~s}$ preceding the onset of hindpaw withdrawal to $4 \mathrm{~s}$ after the onset of pinch stimulation were derived by calculating $(F$ $-F 0) / F 0$ for each pinch stimulation, where $F 0$ is the median $\mathrm{Ca}^{2+}$ transient at $2 \mathrm{~s}$ preceding pinch stimulation to its onset. The $\Delta F / F$ values of all pinch stimuli were then averaged and plotted with a shaded area indicating the SEM.

In some experiments, in vivo $\mathrm{Ca}^{2+}$ imaging with micro-endoscopy was used ${ }^{45}$. Adult mice were anaesthetized with sodium pentobarbital $(1 \%, 40 \mathrm{mg} / \mathrm{kg}$, i.p.) and treated with dexamethasone $(0.2 \mathrm{mg} / \mathrm{kg}$, s.c. $)$ to prevent brain swelling and inflammation. During the procedures, a piece of skull ( $1 \mathrm{~mm}$ in diameter) above the LPBN was removed after high-speed dental drilling. AAV-hSyn-DIO-

GCaMP7s virus was injected into the LPBN (AP, $5.02 \mathrm{~mm}$; ML, $\pm 1.25 \mathrm{~mm}$; DV, $3.27 \mathrm{~mm})$ in VgluT2-ires-Cre mice. A ProView implant lens probe $(\sim 6.1 \mathrm{~mm}$ length, $0.5 \mathrm{~mm}$ diameter; Inscopix, \#1050-002211) was inserted into the brain and targeted to $\sim 50-100 \mu \mathrm{m}$ above the injection. Three weeks after injection, the baseplate of a miniaturized integrated fluorescence microscope (Inscopix, \#1050002192) was fixed on top of the microendoscope in well-labeled animals. $\mathrm{Ca}^{2+}$ was imaged and analyzed as in our previous reports ${ }^{45}$.

Three to four days after baseplate surgery, animals were habituated to the attachment of the microscope and environment for 1 day. $\mathrm{Ca}^{2+}$ was then imaged in freely-moving mice with von Frey filaments $(0.4 \mathrm{~g}$ and $1.0 \mathrm{~g})$ or forceps pinch to the hindpaw. $\mathrm{CPN}$ was then performed and $\mathrm{Ca}^{2+}$ was imaged $-\mathrm{n}$ the same animals 7 days after CPN. Each imaging session included 4 blocks: 20 min habituation and $4 \mathrm{~min}$ of free movement followed by 8-12 trials (trial interval, $10 \mathrm{~s}$ ) of von Frey and pinch stimulation.

$\mathrm{Ca}^{2+}$ was imaged in freely-moving mice using the head-attached microscope as in our previous reports ${ }^{45}$ (Inscopix; LED power, $0.6-1.0 \mathrm{~mW}$; camera resolution, $1440 \times 1080$ pixels). Mouse behavior was recorded with a video camera (Canon) synchronized with $\mathrm{Ca}^{2+}$ imaging using the trigger-out signal from nVista $\mathrm{HD}^{45}$. $\mathrm{Ca}^{2+}$ imaging videos were analyzed and neuronal signals were detected using Imaging Data Processing (Inscopix) and custom-written scripts in MatLab following published algorithms (PCA-ICA) ${ }^{45,84,85}$.

For each neuron, the amplitude of each $\mathrm{Ca}^{2+}$ peak was normalized by the standard deviation of the whole $\mathrm{Ca}^{2+}$ trace. To visualize the activity patterns of neuron during stimulation, the active event traces of each excited LPBN neuron were aligned by the time when the von Frey filament or pinch was applied to the plantar surface of the left hindpaw. The resulting traces from all LPBN neurons were sorted by their peak activation time during the window and displayed in temporal raster plots. To quantify the difference in activity between pre-CPN and post-CPN conditions, we first calculated the trial-averaged activity of LPBN neurons in response to different sensory stimuli, then compared the responses between pre-CPN and post-CPN conditions within $1 \mathrm{~s}$ of stimulation. A significant increase in activity of LPBN neurons in response to von Frey filaments and pinch was found after CPN.

Open field test. The open field arena was a square box $(50 \times 50 \mathrm{~cm})$ with opaque Plexiglas walls. Each test mouse was placed in the center of the box and recorded by a camera attached to a computer. The movement was automatically tracked and analyzed by ANY-maze software. The total distance traveled and time spent in the center area $(25 \times 25 \mathrm{~cm})$ were measured. To assess the effect of optogenetic activation or suppression of LPBN neurons on locomotor activity, mice were tested for a 15-min session consisting of 5-min Pre, 5-min Light, and 5-min Post periods. Blue $(473 \mathrm{~nm}, 5 \mathrm{~ms}, 5-10 \mathrm{~mW})$ or yellow $(589 \mathrm{~nm}, 1 \mathrm{~Hz}, 999 \mathrm{~ms}, 10 \mathrm{~mW})$ laser light was delivered bilaterally during the light On phase. The box was cleaned with $70 \%$ ethanol after each trial. The immobility time and time spent in the center area were automatically analyzed by ANY-maze software 5.3.

Real-time place avoidance (RTPA) test. Mice were placed in a custom-made $50 \times 27 \times 30 \mathrm{~cm}$ two-chamber apparatus made with opaque acrylic Plexiglas that had distinct stripe patterns. Each mouse was placed in the center and allowed to explore both chambers without light stimulation for $10 \mathrm{~min}$. After exploration, the mouse indicated a small preference for one of the two chambers. Subsequently, blue light stimulation $(20 \mathrm{~Hz}, 5$-ms pulse width, $\sim 5 \mathrm{~mW}$ for CaMKII $\alpha$-positive neurons and VgluT2-positive neurons) was delivered whenever the mouse entered or stayed in the preferred chamber, and the light was turned off when the mouse moved to the other chamber (stimulation phase) for $10 \mathrm{~min}$. Finally, the mouse was allowed to freely explore both chambers without blue light stimulation for $10 \mathrm{~min}$. The RTPA location plots and total time on the stimulated side were recorded and counted with the ANY-maze software 5.3 via a webcam (Logitech web-camera) $)^{41,42}$.

Real-time place preference (RTPP) test. Mice were placed in a custom-made $50 \times 27 \times 30 \mathrm{~cm}$ two-chamber apparatus made with opaque acrylic Plexiglas that had distinct stripe patterns. Each mouse was placed in the center of the apparatus and allowed to explore both chambers without light stimulation for $10 \mathrm{~min}$. After exploration, the mouse normally showed a small preference for one of the two sides. Subsequently, blue light stimulation $(40 \mathrm{~Hz}, 5$-ms pulse width, $\sim 5 \mathrm{~mW})$ was delivered whenever the mouse entered or stayed in the non-preferred chamber, and the light was turned off when the mouse moved to the other chamber (stimulation phase) for $10 \mathrm{~min}$. Finally, the mouse was allowed to freely explore both chambers without blue light stimulation for $10 \mathrm{~min}$. The RTPP location plots and total time on the stimulated side were recorded and counted with the ANY-maze software 5.3 via a webcam (Logitech web-camera) $)^{20,41}$.

Conditioned place aversion (CPA) test. To validate the activation of glutamatergic neurons with (DREADD-hM3Dq), AAV-hSyn-DIO-hM3Dq-mCherry (80 $\mathrm{nl} /$ side) and AAV-EF1 $\alpha$-DIO-mCherry virus ( $80 \mathrm{nl} /$ side) were injected into VgluT2-ires-Cre mice. Three weeks later, the mice were subjected to a 2-chamber classical conditioned place aversion (CPA) test. Each mouse was first habituated to the chamber on days 1 and 2 (baseline) for 30 min each day. On day 3, each mouse was placed in the center of the box and allowed to explore both chambers without stimulation (screening test) for $10 \mathrm{~min}$. Generally, after exploration, the mouse showed a small preference for one of the chambers. On days 4-6, each mouse was confined to the preferred chamber with saline injection (i.p.) for $30 \mathrm{~min}$ in the morning, and $4 \mathrm{~h}$ later confined to the non-preferred chamber with $\mathrm{CNO}$ injection $(1.5 \mathrm{mg} / \mathrm{kg}$, i.p.) for $30 \mathrm{~min}$ in the afternoon. On the final day (day 7$)$, each mouse was allowed to explore both chambers with saline or CNO for $10 \mathrm{~min}$, and its behavior was recorded and counted with the ANY-maze software 5.3 via a webcam (Logitech web-camera). 
Immunohistochemical staining. Staining with c-Fos was used to assess the effect of in vivo optogenetic and pharmacogenetic manipulations on neuronal activity in the LPBN. Mice were stimulated with blue or yellow light for $10 \mathrm{~min}$. They were then transcardially perfused with $0.1 \mathrm{M}$ PBS and $4 \%$ paraformaldehyde in PBS about $60-90$ min after light stimulation. The brain was then removed and fixed in $4 \%$ paraformaldehyde buffer at $4{ }^{\circ} \mathrm{C}$ overnight. After fixation, the brain was cryoprotected in $30 \%$ sucrose $(\mathrm{wt} / \mathrm{vol})$ at $4{ }^{\circ} \mathrm{C}$. Free-floating sections $(35-40 \mu \mathrm{m})$ cut on a cryostat (Leica CM 1950) were used for immunohistochemical staining, except for that implanted with optical fibers or cannulae, which were mounted on slides immediately after sectioning. After rinsing with ice-cold methanol (10 min) and blocking with $10 \%$ (wt/vol) normal bovine serum for $1 \mathrm{~h}$ at room temperature, the sections were incubated $\left(12-24 \mathrm{~h}\right.$ at $\left.4{ }^{\circ} \mathrm{C}\right)$ with the primary antibodies anti-cFos (1:500, Guinea pig, SYSY), anti-c-Fos (1:1000, rabbit, SYSY), anti-CaMKIIa (1:1000, rabbit, Abcam), and anti-vGAT (1:800, mouse, SYSY). After rinsing, the sections were incubated with fluorophore-conjugated secondary antibodies for $2 \mathrm{~h}$ at room temperature (1:1000, anti-Guinea pig Alexa Fluor 488, anti-rabbit Cy3, anti-Guinea pig Cy3, anti-rabbit Alexa Fluor 488, Jackson; 1:1000, anti-mouse Alexa Fluor 488, Invitrogen). The antibodies were diluted in phosphate-buffered saline containing 5\% BSA. For c-Fos quantification, mice were exposed to blue $(473 \mathrm{~nm}, 20 \mathrm{~Hz} / 40 \mathrm{~Hz}, 5$-ms pulse width, $5 \mathrm{~mW})$ or yellow $(589 \mathrm{~nm}, 1 \mathrm{~Hz}, 999-\mathrm{ms}$ pulse width, $10 \mathrm{~mW}$ ) laser for $10 \mathrm{~min}$, perfused for $60 \mathrm{~min}$, and sections were cut. After anti-Fos immunohistochemistry, nuclei were stained with DAPI, and confocal images were captured on an Olympus FV-1200 microscope. Cell counting was carried out manually using Image J-win64.

RNAscope in situ hybridization. RNAscope Fluorescent Multiplex Assays with the GAD1 probe were used to determine the overlap of different neuron types in the LPBN. Brain sections from VgluT2-ires-Cre mice injected with rabies virus (RV-EvnA-DsRed) in the LPBN were cut at $40 \mu \mathrm{m}$ on a cryostat (Leica CM 1950), and collected into DepC-PBS. Floating sections were mounted on slides, heated at $60{ }^{\circ} \mathrm{C}$ for $2 \mathrm{~h}$ and kept at $-80^{\circ} \mathrm{C}$ before experiments. Slides were treated thrice with ethanol ( $5 \mathrm{~min}$ each time) and air-dried at room temperature. Then, the slides were pretreated with hydrogen peroxide at room temperature for $10 \mathrm{~min}$ and washed twice in DepC-PBS ( 2 min each time). Protease digestion was performed in a $40^{\circ} \mathrm{C}$ HybEZ oven for 15 min. After washing in DepC-PBS for 3 min and rinsing in DepC-ddH $\mathrm{H}_{2} \mathrm{O}$, slides were hybridized with pre-warmed GAD1 probe, Slc17a6 probe (VgluT2), positive control probe (peptidylprolyl isomerase B, Ppib), or negative control probe [dihydrodipicolinate reductase (dapB) gene] in the $40{ }^{\circ} \mathrm{C}$ HybEZ oven for $2 \mathrm{~h}$. The signal amplification fluorescent label was TSA-based. Anti-DsRed staining was performed after the RNAscope staining to define DsRed ${ }^{+}$ neurons. RNAscope multiplex fluorescent reagent kit v2 and appropriately designed probes (ACDBio Inc.) were used. The probes were alternated across all sections to ensure that one posterior section and one anterior section from each region was analyzed with each probe type. Poorly stained slides were not analyzed.

Image acquisition and quantification. Samples were imaged on a Olympus FV1200 laser scanning confocal microscope. All immunohistochemical staining samples were imaged at $\times 10$ resolution. In situ samples were imaged at $\times 20$ resolution at three $z$-positions. All $z$-positions for each slice were merged into a single image in Adobe Photoshop CC for quantification. The captured neurons and Fos-expressing neurons in all immunohistochemical and in situ hybridization experiments were manually counted, and percentages were calculated for each animal before averaging the percentages across animals. To evaluate the relative marker expression (Fig. 1a-h and Fig. $7 \mathrm{~g}-\mathrm{j}$ ), we summed the cell counts across brain sections from 5 or 6 mice.

Electrophysiological recording in acute brain slices. For whole-cell recordings, each mouse was anesthetized with isoflurane and the brain dissected rapidly and immersed in ice-cold oxygenated $\left(95 \% \mathrm{O}_{2} / 5 \% \mathrm{CO}_{2}\right)$ artificial cerebrospinal fluid (ACSF) (in mM: $124 \mathrm{NaCl}, 2 \mathrm{KCl}, 2 \mathrm{MgSO}_{4}, 1.25 \mathrm{KH}_{2} \mathrm{PO}_{4}, 2 \mathrm{CaCl}_{2}, 26 \mathrm{NaHCO}_{3}$, 10 D-glucose, $\mathrm{pH} 7.4,300 \mathrm{mOsm})$. Coronal slices $(300 \mu \mathrm{m})$ containing the LPBN complex were cut on a vibratome (Microm HM $650 \mathrm{~V}$ ), and then allowed to recover in ACSF. Slices were placed on glass coverslips and submerged in a recording chamber. Whole-cell voltage-clamp recordings were amplified with Multiclamp 700B (filtered at $2 \mathrm{kHz}$ ), digitized with Digidata $1440 \mathrm{~A}(5 \mathrm{kHz})$, and recorded using pClamp 10.4 software (RRID: SCR_011323). Borosilicate glass pipettes (Sutter instruments) (3-5 M 2 ) were pulled on a horizontal pipette puller (P97, Sutter Instruments) and filled with artificial intracellular fluid (in mM: 130 CsCl, 1 EGTA, $0.5 \mathrm{CaCl}_{2}, 2 \mathrm{MgCl}_{2}$, 2 QX-314, 2 ATP-Na, 0.4 GTP-Na; pH 7.3, 285-290 mOsm). Both mCherry ${ }^{+}$(glutamatergic) and eYFP ${ }^{+}$(GABAergic) LPBN neurons were visualized under the Olympus microscope. Neurons, axons, and synaptic terminals expressing ChR2 were activated by $10-\mathrm{ms}$ flashes of blue light $(473 \mathrm{~nm}, 1 \mathrm{~Hz})$ from a single-wavelength laser system (Newdoon Inc., Hangzhou, China). The blue light was focused on the aperture of the microscope objective, producing a wide-field exposure around the recorded cell. To confirm that postsynaptic currents were monosynaptic, eIPSC signals were recorded at $-70 \mathrm{mV}$ in ACSF containing tetrodotoxin (TTX; $1 \mu \mathrm{M}$ ), followed by a combination of TTX and 4-aminopyridine (4-AP; $100 \mu \mathrm{M}$ ), and finally by a combination of TTX, 4-AP, and bicuculline $(10 \mu \mathrm{M})$.
To establish the efficiency of ChR2 expression, blue laser light (473 nm, 1, 5, and $20 \mathrm{~Hz}$ ) was delivered to a neuron expressing ChR2 and the evoked time-locked membrane depolarization and action potentials were recorded. Whole-cell patchclamp recordings were made at room temperature. The internal solution contained (in $\mathrm{mM}$ ): $75 \mathrm{CsCl}, 75 \mathrm{CsF}, 2.5 \mathrm{MgCl}_{2}, 5 \mathrm{HEPES}, 2.5 \mathrm{EGTA}, 14$ creatine phosphateTris, $4 \mathrm{Mg}$-ATP, $0.3 \mathrm{GTP}$-Tris. Blue laser light $(473 \mathrm{~nm}, 1,5$, and $20 \mathrm{~Hz})$ was delivered to the recorded neuron and used to activate glutamatergic neurons (eYFP) in the LPBN. Signals were filtered at $2 \mathrm{kHz}$, sampled at $10 \mathrm{kHz}$, and analyzed using Igor pro software.

In some experiments, current-clamp recordings were performed using glass electrodes (3-5 M $\Omega$ ) containing (in $\mathrm{mM}$ ) $135 \mathrm{~K}$-gluconate, $6 \mathrm{NaCl}, 10 \mathrm{HEPES}, 0.5$ EGTA, $10 \mathrm{Na}_{2}$-phosphocreatine, $4 \mathrm{Mg}$-ATP, $0.3 \mathrm{Na}$-GTP (pH 7.2 adjusted with $\mathrm{KOH}$; 290-300 mOsm).

All electrophysiological data were analyzed off-line using the Neuromatic package (Think Random) in Igor Pro software (WaveMetrics). Off-line analysis was performed by averaging five traces. Light-evoked IPSC peak amplitude, onset latency, time to peak, rise time, and decay time were analyzed. The onset latency of the light-evoked IPSCs was defined as the time from the onset of the stimulus to the first measurable deflection of the potential from baseline. After recording, the slices were fixed with $4 \%$ paraformaldehyde in PBS for immunohistochemical staining.

Statistical analysis. All experiments and data analyses were conducted blinded, including the immunohistochemistry, electrophysiology and behavioral analyses. Data were analyzed with Graphpad Prism v.6.0, Image J1.48V, Image J-win64, Olympus FV10-ASW 4.0a Viewer, Adobe Illustrator CS6 (version 16), Adobe Photoshop CC 2017, Microsoft office 2013, Adobe Acrobat Pro 2017, IBM SPSS_26.0, and MATLAB R2016a software.

Since our data were continuous variables, parametric statistics were used. The homogeneity of variance was assessed by Levene's test of Equality of Error Variances. Square-root transformation or logarithmic transformation was performed for data that did not meet the homogeneity of variance test. Behavioral data were analyzed by one-way or two-way analysis of variance (ANOVA) followed by Bonferroni's test for multiple comparisons, and the unpaired $t$-test for twogroup comparisons. Imaging of calcium activity data were analyzed by Wilcoxon Signed Rank Test. For immunofluorescence analysis, data were analyzed using oneway ANOVA or unpaired Student's $t$-tests. Statistical significance was accepted at the level of $P<0.05$ with asterisks in figures denoting $P$ values as follows: ${ }^{*} P<0.05$, $* * P<0.01, * * * P<0.001, * * * * P<0.0001$. All data are presented as the mean \pm standard error of the mean (SEM). No statistical methods were used to predetermine sample sizes, but our sample sizes are similar to those reported in previous publications ${ }^{41,43}$. Supplementary Table 1 provided statistical data for all figures.

Reproducibility. Experiments were repeated independently with similar results at least three times. Micrographic images presented in figures are representative ones from experiments repeated independently: Fig. 1a, b (four times), Fig. 1e (three times), Fig. $1 \mathrm{~g}$ (three times), Fig. 6a (five times), Fig. $7 \mathrm{~g}$ (four times), Fig. $8 \mathrm{~b}$ (three times), Fig. 8d (four times), Fig. 9j (three times).

Reporting summary. Further information on research design is available in the Nature Research Reporting Summary linked to this article.

\section{Data availability}

Source data are provided with this paper. All data are contained in the main text and the supplementary materials, and are available from the corresponding author upon reasonable request. The source data underlying Figs. $1 \mathrm{c}, \mathrm{d}, \mathrm{f}, \mathrm{h}, \mathrm{k}, \mathrm{n} ; 2 \mathrm{~d}, \mathrm{e}, \mathrm{h}, \mathrm{j} ; 3 \mathrm{~b}-\mathrm{e}, \mathrm{h} ; \mathrm{4b}$, c, f, g, j-m; 5b, c, e, f, h-k; 6b, c, e, g, i-l, n; 7b, c, f, h, i, j; 8f, g, j, k; 9b, e, g, i, k and Supplementary Figs. 9c; 2f, g; 3b, d; 5b, c, e and 6b, e are provided as a Source Data file. Allen Brain Atlas (http://www.brain-map.org).

Received: 28 February 2020; Accepted: 28 October 2020; Published online: 25 November 2020

\section{References}

1. Costigan, M., Scholz, J. \& Woolf, C. J. Neuropathic pain: a maladaptive response of the nervous system to damage. Annu Rev. Neurosci. 32, 1-32 (2009).

2. Colloca, L. et al. Neuropathic pain. Nat. Rev. Dis. Prim. 3, 17002 (2017).

3. Basbaum, A. I., Bautista, D. M., Scherrer, G. \& Julius, D. Cellular and molecular mechanisms of pain. Cell 139, 267-284 (2009).

4. Bushnell, M. C., Ceko, M. \& Low, L. A. Cognitive and emotional control of pain and its disruption in chronic pain. Nat. Rev. Neurosci. 14, 502-511 (2013). 
5. Kuner, R. \& Flor, H. Structural plasticity and reorganisation in chronic pain. Nat. Rev. Neurosci. 18, 20-30 (2016).

6. Woolf, C. J. \& Salter, M. W. Neuroscience-neuronal plasticity: increasing the gain in pain. Science 288, 1765-1768 (2000).

7. Kuner, R. Central mechanisms of pathological pain. Nat. Med. 16, 1258-1266 (2010).

8. Cichon, J., Blanck, T. J. J., Gan, W. B. \& Yang, G. Activation of cortical somatostatin interneurons prevents the development of neuropathic pain. Nat. Neurosci. 20, 1122-1132 (2017).

9. Zhuo, M. Neural mechanisms underlying anxiety-chronic pain interactions. Trends Neurosci. 39, 136-145 (2016).

10. Melzack, R. \& Wall, P. D. Pain mechanisms: a new theory. Science 150, 971-979 (1965).

11. Peirs, C. et al. Dorsal horn circuits for persistent mechanical pain. Neuron 87, 797-812 (2015).

12. Duan, B., Cheng, L. \& Ma, Q. Spinal circuits transmitting mechanical pain and itch. Neurosci. Bull. 34, 186-193 (2018).

13. Saab, C. Y. Pain-related changes in the brain: diagnostic and therapeutic potentials. Trends Neurosci. 35, 629-637 (2012).

14. Navratilova, E. \& Porreca, F. Reward and motivation in pain and pain relief. Nat. Neurosci. 17, 1304-1312 (2014)

15. Davern, P. J. A role for the lateral parabrachial nucleus in cardiovascular function and fluid homeostasis. Front. Physiol. 5, 436 (2014).

16. Hermanson, O. \& Blomqvist, A. Subnuclear localization of FOS-like immunoreactivity in the parabrachial nucleus after orofacial nociceptive stimulation of the awake rat. The. J. Comp. Neurol. 387, 114-123 (1997).

17. Swanson, L. W. \& Sawchenko, P. E. Paraventricular nucleus: a site for the integration of neuroendocrine and autonomic mechanisms. Neuroendocrinology 31, 410-417 (1980).

18. Gauriau, C. \& Bernard, J. F. Pain pathways and parabrachial circuits in the rat. Exp. Physiol. 87, 251-258 (2002).

19. Roeder, Z. et al. Parabrachial complex links pain transmission to descending pain modulation. Pain 157, 2697-2708 (2016).

20. Rodriguez, E. et al. A craniofacial-specific monosynaptic circuit enables heightened affective pain. Nat. Neurosci. 20, 1734-1743 (2017).

21. Al-Khater, K. M. \& Todd, A. J. Collateral projections of neurons in laminae I, III, and IV of rat spinal cord to thalamus, periaqueductal gray matter, and lateral parabrachial area. J. Comp. Neurol. 515, 629-646 (2009).

22. Bernard, J. F., Huang, G. F. \& Besson, J. M. The parabrachial area: electrophysiological evidence for an involvement in visceral nociceptive processes. J. Neurophysiol. 71, 1646-1660 (1994).

23. Bernard, J. F., Bester, H. \& Besson, J. M. Involvement of the spino-parabrachio -amygdaloid and -hypothalamic pathways in the autonomic and affective emotional aspects of pain. Prog. Brain Res. 107, 243-255 (1996).

24. Grigson, P. S., Scalera, G., Reilly, S. \& Norgren, R. The parabrachial nucleus is essential for acquisition of a conditioned odor aversion in rats. Behav. Neurosci. 112, 1104-1113 (1998)

25. Rupprecht, R. \& Di Benedetto, B. Extracellular signal-regulated kinases: a role for mood disorders and the emotional component of pain? Biol. Psychiatry 81, 639-641 (2017).

26. Wilson, T. D. et al. Dual and opposing functions of the central amygdala in the modulation of pain. Cell Rep. 29, 332-346 e335 (2019).

27. Li, J. N. \& Sheets, P. L. Spared nerve injury differentially alters parabrachial monosynaptic excitatory inputs to molecularly specific neurons in distinct subregions of the central amygdala. Pain 161, 166-176 (2020).

28. Raver, C. et al. An amygdalo-parabrachial pathway regulates pain perception and chronic pain. J. Neurosci. 40, 3424-3442 (2020).

29. Chiang, M. C. et al. Divergent neural pathways emanating from the lateral parabrachial nucleus mediate distinct components of the pain response. Neuron 106, 927-939 e925 (2020).

30. Carter, M. E., Soden, M. E., Zweifel, L. S. \& Palmiter, R. D. Genetic identification of a neural circuit that suppresses appetite. Nature 503, 111-114 (2013).

31. Han, S., Soleiman, M. T., Soden, M. E., Zweifel, L. S. \& Palmiter, R. D. Elucidating an affective pain circuit that creates a threat memory. Cell 162, 363-374 (2015).

32. $\mathrm{Mu}$, D. et al. A central neural circuit for itch sensation. Science 357, 695-698 (2017).

33. Alhadeff, A. L. et al. A neural circuit for the suppression of pain by a competing need state. Cell 173, 140-152 el15 (2018)

34. Campos, C. A. et al. Cancer-induced anorexia and malaise are mediated by CGRP neurons in the parabrachial nucleus. Nat. Neurosci. 20, 934-942 (2017).

35. Missig, G. et al. Parabrachial nucleus (PBn) pituitary adenylate cyclase activating polypeptide (PACAP) signaling in the amygdala: implication for the sensory and behavioral effects of pain. Neuropharmacology 86, 38-48 (2014).

36. Uddin, O. et al. Amplified parabrachial nucleus activity in a rat model of trigeminal neuropathic pain. Neurobiol. pain. 3, 22-30 (2018).
37. Francois, A. et al. A brainstem-spinal cord inhibitory circuit for mechanical pain modulation by GABA and enkephalins. Neuron 93, 822-839 e826 (2017).

38. King, T. et al. Unmasking the tonic-aversive state in neuropathic pain. Nat. Neurosci. 12, 1364-1366 (2009).

39. Vadakkan, K. I., Jia, Y. H. \& Zhuo, M. A behavioral model of neuropathic pain induced by ligation of the common peroneal nerve in mice. J. Pain. 6, 747-756 (2005).

40. Gunaydin, L. A. et al. Natural neural projection dynamics underlying social behavior. Cell 157, 1535-1551 (2014).

41. Yang, $\mathrm{H}$. et al. Laterodorsal tegmentum interneuron subtypes oppositely regulate olfactory cue-induced innate fear. Nat. Neurosci. 19, 283-289 (2016).

42. Stamatakis, A. M. \& Stuber, G. D. Activation of lateral habenula inputs to the ventral midbrain promotes behavioral avoidance. Nat. Neurosci. 15, 1105 (2012).

43. Sun, L. et al. Microglial cathepsin B contributes to the initiation of peripheral inflammation-induced chronic pain. J. Neurosci. 32, 11330-11342 (2012).

44. $\mathrm{Li}, \mathrm{X}$. et al. Skin suturing and cortical surface viral infusion improves imaging of neuronal ensemble activity with head-mounted miniature microscopes. $J$. Neurosci. Methods 291, 238-248 (2017).

45. Liu, Y. et al. Touch and tactile neuropathic pain sensitivity are set by corticospinal projections. Nature 561, 547-550 (2018)

46. Pitcher, G. M., Ritchie, J. \& Henry, J. L. Paw withdrawal threshold in the von Frey hair test is influenced by the surface on which the rat stands. J. Neurosci. Methods 87, 185-193 (1999).

47. Deyama, S. et al. Inhibition of glutamatergic transmission by morphine in the basolateral amygdaloid nucleus reduces pain-induced aversion. Neurosci. Res. 59, 199-204 (2007).

48. Johansen, J. P. \& Fields, H. L. Glutamatergic activation of anterior cingulate cortex produces an aversive teaching signal. Nat. Neurosci. 7 , 398-403 (2004).

49. Malfliet, A. et al. Brain changes associated with cognitive and emotional factors in chronic pain: a systematic review. Eur. J. Pain. 21, 769-786 (2017)

50. Baastrup, C., Jensen, T. S. \& Finnerup, N. B. Pregabalin attenuates place escape/avoidance behavior in a rat model of spinal cord injury. Brain Res. 1370, 129-135 (2011)

51. LaBuda, C. J. \& Fuchs, P. N. A behavioral test paradigm to measure the aversive quality of inflammatory and neuropathic pain in rats. Exp. Neurol. 163, 490-494 (2000).

52. Zhang, Z. Z. et al. Role of prelimbic GABAergic circuits in sensory and emotional aspects of neuropathic pain. Cell Rep. 12, 752-759 (2015).

53. Scopinho, A. A., Resstel, L. B. \& Correa, F. M. alpha(1)-Adrenoceptors in the lateral septal area modulate food intake behaviour in rats. Br. J. Pharmacol. 155, 752-756 (2008).

54. Wu, Q., Boyle, M. P. \& Palmiter, R. D. Loss of GABAergic signaling by AgRP neurons to the parabrachial nucleus leads to starvation. Cell 137, 1225-1234 (2009).

55. Armbruster, B. N., Li, X., Pausch, M. H., Herlitze, S. \& Roth, B. L. Evolving the lock to fit the key to create a family of $\mathrm{G}$ protein-coupled receptors potently activated by an inert ligand. Proc. Natl Acad. Sci. USA 104, 5163-5168 (2007)

56. Wietek, J., Broser, M., Krause, B. S. \& Hegemann, P. Identification of a natural green light absorbing chloride conducting channelrhodopsin from Proteomonas sulcata. J. Biol. Chem. 291, 4121-4127 (2016).

57. Zhang, J. M. et al. ATP released by astrocytes mediates glutamatergic activitydependent heterosynaptic suppression. Neuron 40, 971-982 (2003).

58. Campos, C. A., Bowen, A. J., Roman, C. W. \& Palmiter, R. D. Encoding of danger by parabrachial CGRP neurons. Nature 555, 617-622 (2018).

59. Barik, A., Thompson, J. H., Seltzer, M., Ghitani, N. \& Chesler, A. T. A brainstem-spinal circuit controlling nocifensive behavior. Neuron 100, 1491-1503 e1493 (2018).

60. Latremoliere, A. \& Woolf, C. J. Central sensitization: a generator of pain hypersensitivity by central neural plasticity. J. Pain. 10, 895-926 (2009).

61. Fuchs, P. N., Peng, Y. B., Boyette-Davis, J. A. \& Uhelski, M. L. The anterior cingulate cortex and pain processing. Front. Integr. Neurosci. 8, 35 (2014).

62. Polgar, E., Durrieux, C., Hughes, D. I. \& Todd, A. J. A quantitative study of inhibitory interneurons in laminae I-III of the mouse spinal dorsal horn. PLoS ONE 8, e78309 (2013).

63. Echeverry, S. et al. Spinal microglia are required for long-term maintenance of neuropathic pain. Pain 158, 1792-1801 (2017).

64. Kawasaki, Y. et al. Distinct roles of matrix metalloproteases in the early- and late-phase development of neuropathic pain. Nat. Med. 14, 331-336 (2008).

65. Chen, G. et al. Connexin-43 induces chemokine release from spinal cord astrocytes to maintain late-phase neuropathic pain in mice. Brain 137, 2193-2209 (2014).

66. Duan, B. et al. Identification of spinal circuits transmitting and gating mechanical pain. Cell 159, 1417-1432 (2014).

67. Treede, R. D. Gain control mechanisms in the nociceptive system. Pain 157, 1199-1204 (2016) 
68. Moore, K. A. et al. Partial peripheral nerve injury promotes a selective loss of GABAergic inhibition in the superficial dorsal horn of the spinal cord. $J$. Neurosci. 22, 6724-6731 (2002).

69. Coull, J. A. et al. BDNF from microglia causes the shift in neuronal anion gradient underlying neuropathic pain. Nature 438, 1017-1021 (2005).

70. Kawasaki, Y., Zhang, L., Cheng, J. K. \& Ji, R. R. Cytokine mechanisms of central sensitization: distinct and overlapping role of interleukin-1beta, interleukin-6, and tumor necrosis factor-alpha in regulating synaptic and neuronal activity in the superficial spinal cord. J. Neurosci. 28, 5189-5194 (2008).

71. Halievski, K., Ghazisaeidi, S. \& Salter, M.W. Sex-dependent mechanisms of chronic pain: a focus on microglia and P2X4R. J. Pharmacol. Experiment. Therapeut. 375, 202-209 (2020).

72. Jones, A. F. \& Sheets, P. L. Sex-specific disruption of distinct mPFC inhibitory neurons in spared-nerve injury model of neuropathic pain. Cell Rep. 31, 107729 (2020)

73. Chen, G., Luo, X., Qadri, M. Y., Berta, T. \& Ji, R. R. Sex-dependent glial signaling in pathological pain: distinct roles of spinal microglia and astrocytes. Neurosci. Bull. 34, 98-108 (2018).

74. Jhamandas, J. H., Petrov, T., Harris, K. H., Vu, T. \& Krukoff, T. L. Parabrachial nucleus projection to the amygdala in the rat: Electrophysiological and anatomical observations. Brain Res. Bull. 39, 115-126 (1996).

75. Schwaber, J. S., Sternini, C., Brecha, N. C., Rogers, W. T. \& Card, J. P. Neurons containing calcitonin gene-related peptide in the parabrachial nucleus project to the central nucleus of the amygdala. J. Comp. Neurol. 270, 416-426 (1988). 398-419.

76. Rouwette, T., Vanelderen, P., Roubos, E. W., Kozicz, T. \& Vissers, K. The amygdala, a relay station for switching on and off pain. Eur. J. Pain. 16, 782-792 (2012).

77. Huang, J. et al. A neuronal circuit for activating descending modulation of neuropathic pain. Nat. Neurosci. 22, 1659-1668 (2019).

78. Loeser, J. Five crises in pain management. Revista de la Sociedad Española del Dolor 19, 111-116 (2012).

79. Hayashi, Y. et al. Microglial $\mathrm{Ca}(2+)$-activated $\mathrm{K}(+)$ channels are possible molecular targets for the analgesic effects of S-ketamine on neuropathic pain. J. Neurosci. 31, 17370-17382 (2011).

80. Hargreaves, B. J., Kronfeld, D. S. \& Naylor, J. R. J. Ambient temperature and relative humidity influenced packed cell volume, total plasma protein and other variables in horses during an incremental submaximal field exercise test. Equine Vet. J. 31, 314-318 (1999).

81. Han, Y. et al. Selective activation of cholinergic basal forebrain neurons induces immediate sleep-wake transitions. Curr. Biol. 24, 693-698 (2014).

82. Huang, T. et al. Identifying the pathways required for coping behaviours associated with sustained pain. Nature 565, 86-90 (2019).

83. Li, Y. et al. Hypothalamic circuits for predation and evasion. Neuron 97, 911-924 e915 (2018).

84. Mukamel, E. A., Nimmerjahn, A. \& Schnitzer, M. J. Automated analysis of cellular signals from large-scale calcium imaging data. Neuron $63,747-760$ (2009).

85. Hyvarinen, A. \& Oja, E. Independent component analysis: algorithms and applications. Neural Netw. 13, 411-430 (2000).

\section{Acknowledgements}

We thank Zhen-zhong $\mathrm{Xu}$, and I.C. Bruce for discussions and reading the manuscript. We thank Yu-dong Zhou, Kun Chen, Hong-bin Yang, Hui-Fang Lou, and Li-Ya Zhu for technical support. This work was supported by the National Natural Science Foundation of China (31800880, 81527901, and 81821091), the National Key Research and Development Program (2016YFA0501000 and 2016YFC1306700), Key Realm R\&D Program of Guangdong Province (2019B030335001), the Science and Technology Planning Project of Guangdong Province (2018B030331001), CAMS Innovation Fund for Medical Sciences (2018PT31041; 2019-I2M-5-057), and Fundamental Research Funds for the Central Universities (2019FZA7009).

\section{Author contributions}

Conceptualization, L.S. and S.D.; Methodology, L.S., R.L., Z.G.Z., X.Y.L., and S.D.; Investigation, L.S., R.L., F.G., M.Q.W., X.L.M., K.Y.L., H.S., C.L.X., Y.Y.L.; X.J.L.; Visualization, L.S.; Writing, L.S. and S.D.; Funding Acquisition, L.S. and S.D.; Resources, L.S. and S.D.; Supervision, L.S., Y.Q.Y., Z.C., X.Y.L., and S.D.; Statistical analysis, L.S., M.Y.W

\section{Competing interests}

The authors declare no competing interests.

\section{Additional information}

Supplementary information is available for this paper at https://doi.org/10.1038/s41467020-19767-w.

Correspondence and requests for materials should be addressed to L.S. or S.D.

Peer review information Nature Communications thanks Guo-Gang Xing and the other, anonymous, reviewer(s) for their contribution to the peer review of this work. Peer reviewer reports are available.

Reprints and permission information is available at http://www.nature.com/reprints

Publisher's note Springer Nature remains neutral with regard to jurisdictional claims in published maps and institutional affiliations.

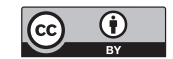

Open Access This article is licensed under a Creative Commons Attribution 4.0 International License, which permits use, sharing, adaptation, distribution and reproduction in any medium or format, as long as you give appropriate credit to the original author(s) and the source, provide a link to the Creative Commons license, and indicate if changes were made. The images or other third party material in this article are included in the article's Creative Commons license, unless indicated otherwise in a credit line to the material. If material is not included in the article's Creative Commons license and your intended use is not permitted by statutory regulation or exceeds the permitted use, you will need to obtain permission directly from the copyright holder. To view a copy of this license, visit http://creativecommons.org/ licenses/by/4.0/.

(C) The Author(s) 2020 